University of New Hampshire

University of New Hampshire Scholars' Repository

Fall 2008

\title{
Underwater tracking of humpback whales (Megaptera novaeangliae) with high-frequency pingers and acoustic recording tags
}

Val E. Schmidt

University of New Hampshire, Durham

Follow this and additional works at: https://scholars.unh.edu/thesis

\section{Recommended Citation}

Schmidt, Val E., "Underwater tracking of humpback whales (Megaptera novaeangliae) with high-frequency pingers and acoustic recording tags" (2008). Master's Theses and Capstones. 397.

https://scholars.unh.edu/thesis/397

This Thesis is brought to you for free and open access by the Student Scholarship at University of New Hampshire Scholars' Repository. It has been accepted for inclusion in Master's Theses and Capstones by an authorized administrator of University of New Hampshire Scholars' Repository. For more information, please contact Scholarly.Communication@unh.edu. 


\title{
UNDERWATER TRACKING OF HUMPBACK WHALES (Megaptera novaeangliae) WITH HIGH-FREQUENCY PINGERS AND ACOUSTIC RECORDING TAGS
}

\author{
BY \\ Val E. Schmidt \\ B.S. Physics University of the South, Sewanee, Tennessee (1994)
}

THESIS

Submitted to the University of New Hampshire

in partial fulfillment of

the requirements for the degree of

Master of Science

in

Ocean Engineering

September 2008 
UMI Number: 1459514

\section{INFORMATION TO USERS}

The quality of this reproduction is dependent upon the quality of the copy submitted. Broken or indistinct print, colored or poor quality illustrations and photographs, print bleed-through, substandard margins, and improper alignment can adversely affect reproduction.

In the unlikely event that the author did not send a complete manuscript and there are missing pages, these will be noted. Also, if unauthorized copyright material had to be removed, a note will indicate the deletion.

(®)

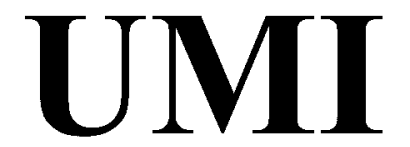

UMI Microform 1459514

Copyright 2008 by ProQuest LLC.

All rights reserved. This microform edition is protected against unauthorized copying under Title 17, United States Code.

ProQuest LLC

789 E. Eisenhower Parkway

PO Box 1346

Ann Arbor, MI 48106-1346 
This thesis has been examined and approved.

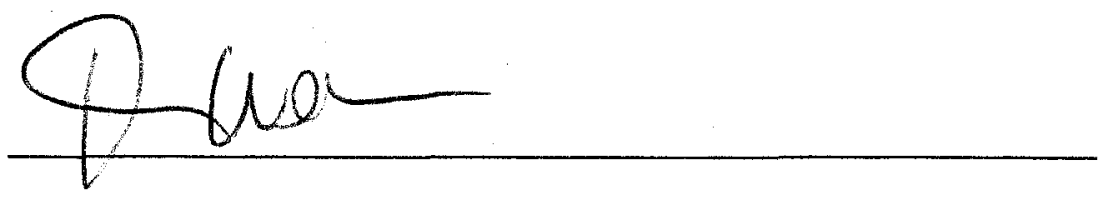

Thesis Director, Thomas C. Weber,

Research Assistant Professor of Ocean Engineering

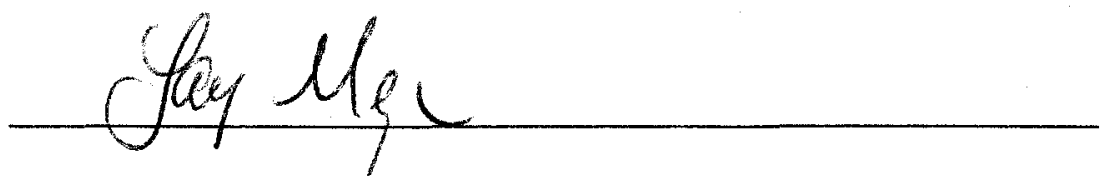

Larry A. Mayer,

Professor of Ocean Engineering

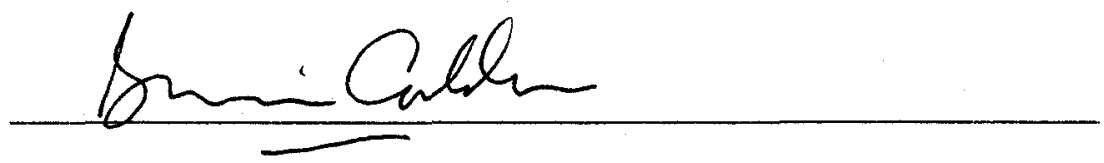

Brian R. Calder,

Research Associate Professor of Ocean Engineering

4 August 2008

Date 


\section{DEDICATION}

I would like to dedicate this work to my family, whose untiring support and encouragement were in every way essential to my success. In particular I would like to thank my best friend and wife, Alice. Her understanding through many long nights and weekends of relentless work and travel has been super-human, and to our daughter, Gracie, whose has endured all this but, has been too young to complain. I am tremendously grateful.

In addition, I would like to offer special thanks to my dear friend Michelle Weirathmueller. Without our endless technical discussions, her thoughtful insight and unerring camaraderie, the quality of this work and my own sanity would have suffered tremendously. 


\section{ACKNOWLEDGEMENTS}

In addition to the paragraph of acknowledgements below, which are taken from the Journal of Ocean Engineering submission verbatim, I would like to give these other special acknowledgments. Tom Weber, my primary technical advisor has spent many hours both in collaboration and in guidance of my work and has been essential to this project. His patience and feedback, along with that of the other committee members, Larry Mayer and Brian Calder, have been invaluable. Tom also created the circuit diagram found in Appendix D from my notes and drawings and is reproduced there with his permission. In addition, the co-authors of the Journal paper (David Wiley, Stellwagen Bank National Marine Sanctuary and Mark Johnson, Woods Hole Oceanographic Institute) have provided guidance and indeed, have made the project possible. Michelle Weirathmueller, my peer and friend, has countless times discussed technical details with me, whose method or meaning I was unable to decipher alone. She has provided much needed moral support and endless encouragement. Finally, I would like to acknowledge my best friend and wife, Alice, whose support at home, including mothering our first child, Gracie, and often working a second job, has been super-human.

The authors would like to acknowledge and thank the entire support staff and whale tagging team at the Stellwagen Bank National Marine Sanctuary, particularly Michael Thompson, whose coordination and logistics support were extraordinary. In addition, Andy McLeod, of the Center for Coastal and Ocean Mapping, provided technical assistance and electronics guidance. Larry Mayer and Brian Calder, also of the Center for Coastal and Ocean Mapping who provided thoughtful feedback on much of the text. Colin Ware produced the visual-fix constrained dead-reckoned whale track in Figure 4-4. William Danforth, of USGS, provided high resolution bathymetry and backscatter on Stellwagen Bank. This project was funded under National Oceanographic and Atmospheric Administration, grant 
NA05NOS4001153. Tagging of whales on Stellwagen Bank was conducted by the staff of the Stellwagen Bank National Marine Sanctuary under NOAA Permit \#775-1600-10. 


\section{TABLE OF CONTENTS}

DEDICATION

ACKNOWLEDGEMENTS iv

LIST OF FIGURES Xii

ABSTRACT

1 INTRODUCTION 1

1.1 Introductory Statement $\ldots \ldots \ldots \ldots \ldots$

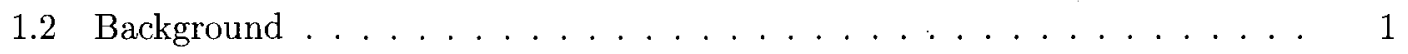

2 WHALE TRACKING SYSTEM DESCRIPTION 6

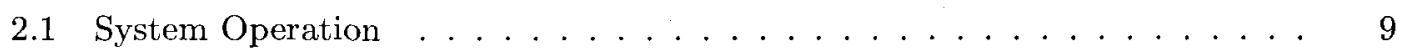

3 DATA PROCESSING

3.1 Generating a Temporal Reference Frame . . . . . . . . . . . . . . . . 13

3.2 Verifying the DTAG Sample Rate and Sample Rate Stability . . . . . . . 14

3.3 Processing the Acoustic Data . . . . . . . . . . . . . . . . . 16

3.4 Calculation of Pinger-to-DTAG Ranges $\ldots \ldots \ldots \ldots \ldots$

3.5 Least Squares Positioning . . . . . . . . . . . . . . . . . . 19

4 EXAMPLE WHALE TRACK 22

4.1 Comparison with Dead-Reckoned Tracks . . . . . . . . . . . . . . . 24

4.2 Whale Track Measurements . . . . . . . . . . . . . . 27

5 CONCLUSION 
$\begin{array}{ll}\text { A STAMP BS2PX CODE } & 38\end{array}$

B MATCHED FILTER BANK GENERATION 44

C PINGER CIRCUIT DIAGRAM 48

D TIME ENCODING ALGORITHM 49

$\begin{array}{ll}\text { BIBLIOGRAPHY } & 51\end{array}$ 


\section{LIST OF FIGURES}

1-1 Stellwagen Bank National Marine Sanctuary is outlined above--east of Boston

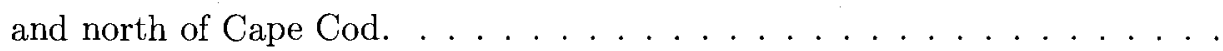

2-1 Small-boats (typically rigid hull inflatable boats, (RHIB)) follow at several hundred meters from the tagged animal. GPS-positioned acoustic sources are deployed from each RHIB. The acoustic sources are recorded on the whale tag, from which one-way travel times and ranges are measured. . . . . . .

2-2 Pinger electronics package with surface-mounted GPS, on/off switch and LED indicator light. The case measures $0.235 \mathrm{~m} \times 0.181 \mathrm{~m} \times 0.146 \mathrm{~m}$. . . .

2-3 Conceptual drawing of the pinger. A Garmin ${ }^{T M}$ GPS provides pinger triggering and positioning. Positions are logged to an SD data card. Pulse width modulated acoustic transmit pulses are generated by the control processor, which are anplified and impedance matched to the transducer at the center

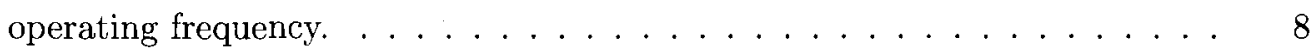

2-4 Flow diagram of the pinger control processor operation. . . . . . . . . . 10

2-5 Spectrograms of all 18 pinger permutations are shown. Each column of three signals are sent from each of the three pingers in one ten second interval. For example, Pingers $\mathrm{A}, \mathrm{B}$ and $\mathrm{C}$ send the first column of three signals, respectively, for seconds 00-09 of every UTC minute. The three signals in the second column are sent from seconds 10-19 of every UTC minute, and so

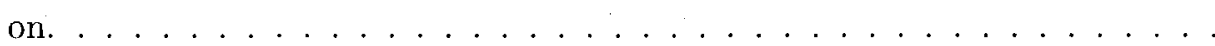


3-1 To measure the DTAG sample rate and determine the need to characterize it as a function of temperature, a controlled measurement was performed by measuring the pinger at a short, known distance. Receive time after transmission is plotted in the upper plot using an assumed sample rate. The true sample rate was found by adjusting the assumed sample rate until the latter portion of the plot ( $>3000$ seconds) is most nearly horizontal, as shown. Comparison of the receive time and temperature plot (below) shows the tag coming to thermal equilibrium. The tag's oscillator varies here just $0.1 \mathrm{ppm}$ over a $6^{\text {circ }}$ temperature change. . . . . . . . . . . 15

3-2 A portion of the raw range measurements from each pinger to the whale are shown. A nominal $1,500 \mathrm{~ms}^{-1}$ sound speed was used to calculate ranges. Circled points indicate those that were retained after a manual cleaning step. Multipath arrivals are frequently evident as parallel lines of data beyond the

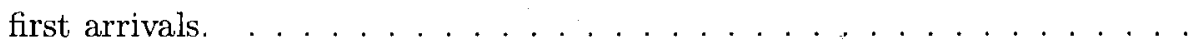

3-3 Individual range measurements from each pinger to the whale are shown. A nominal $1,500 \mathrm{~ms}^{-1}$ sound speed was used to calculate ranges. The apparent discontinuity prior to 22:00 resulted from a repositioning of the pingers when the whale left the immediate feeding area to join other whales several kilometers away at a speed faster than the small-boats could maintain with

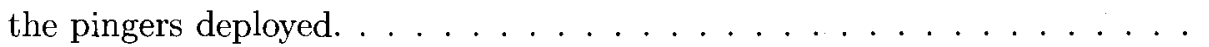


3-4 To disambiguate two-range solution pairs, all fix position information is plotted on a single time-series plot such as the subset of data shown here. For each two-range solution pair, the solution's east/west coordinate is plotted vs time. In addition east/west coordinates from 3-range solutions and visual fixes of the whale while at the surface are plotted. The combined data set allows one to select which of the ambiguous two-range solution pairs is correct using the unambiguous 3-range solutions and the visual fixes as a guide. Outlier 3-range solutions were also omitted. The process is repeated for north/south coordinates and the intersection of the measurement times from each provide the final two-range solution set. All positions are plotted here as meters from the mean value. . . . . . . . . . . . . .

4-1 Plan and perspective views of the acoustically-derived track for the whale "Geometry" shown over local bathymetric data. Shadows of the track data points have been draped on the bathymetry in black for clarity. The perspective view is looking to the North-Northwest from atop Stellwagen Bank. Vertical exaggeration is $10 \mathrm{x}$. The track duration is approximately 80 minutes and the general path of the whale is from left to right. ........

4-2 A portion acoustically measured whale track is shown with dead-reckoned whale track for the same time. Points in both tracks are plotted from the mean of the acoustic data. The dead-reckoned whale track is generated from an initial visual fix approximately 1.6 hours prior to the data shown here, and an assumed $1 \mathrm{~ms}^{-1}$ whale speed. Errors in the dead-reckoned track over this time frame accumulate quickly resulting in a track offset by nearly $2 \mathrm{~km} .25$ 
4-3 The pairs of plots provide a closer comparison of the acoustically derived and dead-reckoned whale tracks shown in Figure 4.1. The left plot in each subfigure shows acoustically derived whale positions, while the right plot shows dead-reckoned track with an assumed constant velocity for the same time as each of the plots on the left. In each plot the whale enters from the east and departs to the west. All positions are measured relative to the mean Eastings and Northings of the acoustically derived positions. . . . . . . .

4-4 An acoustically-measured whale track is compared with a constant-velocity pseudo-track and a pseudo-track whose velocity is constrained by visual sightings while the whale is at the surface. Although the fix-constrained pseudo-track is more geographically accurate, the surface fixes cannot capture changes in whale speed while at depth, resulting in the "stringing out" horizontally of vertical bubble net feeding events. Comparison with the acoustically-measured positions shows that, in this case, the non-constrained pseudo-track captures the whale behavior with better fidelity, although the absolute positioning is poor. . . . . . . . . . . . .

4-5 Example of a straight-line segment used to estimate transit speeds in Figure

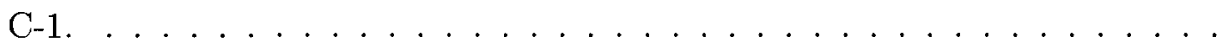

4-6 Transit speed estimations between bubble net feeding events. . . . . . . . .

4-7 Acoustically-measured positions during a bubble net feeding event are plotted, along with a least squares fit of those positions to a circle The one-sigma uncertainty bounds are plotted as dotted lines. Identical methods were used for four other bubble net events whose results are shown in Figure 4-8. . .

4-8 Bubble net radius measurements derived from a least-squares fit to acoustically measured positions. . . . . . . . . . . . . . . . .

4-9 Horizontal mean whale speed estimates during whale rotation and ascension while bubble net feeding. . . . . . . . . . . . . . . . . 
4-10 Three whale dives during feeding events are shown in this track segment. Shadows of each whale position have been draped on the sea floor for clarity. Estimated whale positions intersect the sea floor for the center and right dives, indicating probable actual contact with the sea floor. The whale's movement is from left to right in this image. Vertical exaggeration is 10x. .

B-1 An example waveform of a pinger transmission recorded in the the University of New Hampshire's acoustic test tank. Relative amplitudes of each sub-pulse are a function of both the frequency of the pulse and its relative position within the larger train. . . . . . . . . . . . . . .

B-2 Mean acoustic sub-pulse amplitude weighting factors measured from a full minute of recorded transmissions from one pinger. Since the sub-pulse permutation changes every 10 seconds, 10 amplitude values are averaged to determine each weight. . . . . . . . . . . . .

B-3 The resulting matched filter is shown above a recorded pinger transmission for comparison. . . . . . . . . . . . . . . . . 46

C-1 Pinger circuit diagram. . . . . . . . . . . . . . . . 48

D-1 The method by which time is encoded into each pinger's acoustic signal. . . 


\section{ABSTRACT \\ UNDERWATER TRACKING OF HUMPBACK WHALES (Megaptera novaeangliae) WITH HIGH-FREQUENCY PINGERS AND ACOUSTIC RECORDING TAGS}

by

Val E. Schmidt

University of New Hampshire, September, 2008

A long-baseline acoustic system has been developed for the tracking of humpback whales (Megaptera novaeangliae) that have been tagged with digital acoustic recording devices, or DTAGs, providing quantitative observations of submerged whale behavior. The system includes three acoustic sources deployed from small-boats that follow the whale after the animal has been tagged. Integrated GPS provides positioning and synchronized operation of the sources. Time-encoded signals from the sources are recorded along with whale vocalizations and ambient noise on the whale tag. Time-of-flight measurements, as measured by the tag acoustic data, are converted to range from the whale to each source with a nominal sound speed. A non-linear least-squares solution is then solved for the whale's position. The system is demonstrated with data collected from a tagged animal in the summer of 2007. 


\section{CHAPTER 1}

\section{INTRODUCTION}

\section{$\underline{1.1}$ Introductory Statement}

A pending peer-reviewed submission to the Journal of Oceanic Engineering comprises the bulk of this thesis with only slight modifications to the abstract to meet thesis length requirements. This submission was made with secondary authors, Thomas C. Weber of the University of New Hampshire, David Wiley of the National Oceanic and Atmospheric Administration, and Mark Johnson of Woods Hole Oceanographic Institute. In addition to the article submission, this thesis also contains detailed supplemental information in several appendices.

\section{$\underline{1.2 \quad \text { Background }}$}

Since 2004, the Stellwagen Bank National Marine Sanctuary has facilitated annual summer expeditions to tag humpback whales (Megaptera novaeangliae) that feed in the sanctuary during the summer months $[1,2,3,4]$. Stellwagen Bank is an area north of Cape Cod and east of Boston, with depths ranging from 20 to $200 \mathrm{~m}$ (Figure 1-1). Humpback whales spend their time in the Sanctuary feeding, primarily on sand lance (Ammodytes americanus)[5]. During these expeditions, whales were tagged with Digital Acoustic Recording Tags (DTAGS) developed at Woods Hole Oceanographic Institution [6]. DTAGs have been used in other studies to investigate the kinematics of both sperm and right whales [6] [7], effects of ambient and anthropogenic noise on humpback whale behavior $[8,9]$ and the response of humpback whales to artificially introduced whale calls [10]. In these studies, positioning of the whale has been derived from visual sightings at the surface and dead- 
reckoning while submerged. This work describes a long-baseline (LBL) acoustic system designed to track tagged whales while submerged, providing higher accuracy positioning than the previously used dead-reckoning methods and enabling an increased understanding of whale energetics and behavior.

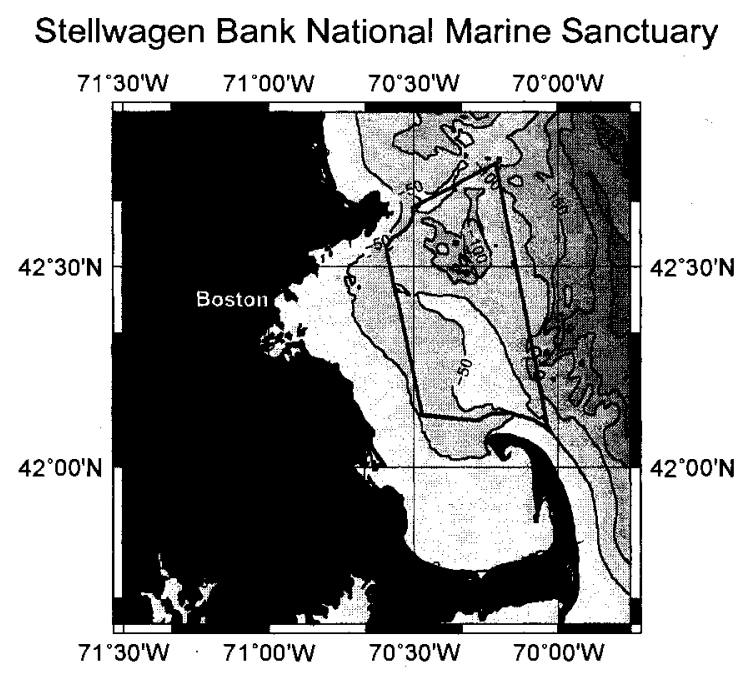

Figure 1-1: Stellwagen Bank National Marine Sanctuary is outlined above-east of Boston and north of Cape Cod.

Common methods of tracking whales include surface sightings, radio-frequency tagging, and both passive and active sonar. Visual sighting and identification of whales at different times of year in different locales remain the standard method of tracking for distances up to thousands of kilometers, and over time intervals from months to years [11]. The advent of radio-frequency microelectronics has improved on visual methods in the form of whale tags that send satellite-received signals for tracking over long distances, or VHF signals for tracking at ranges up to tens of kilometers [11]. However, visual, satellite and VHF tracking methods provide fixes only at the surface. To complement surface fixes, animal tags were developed to measure the depth of the animal over time. Many devices were initially developed to measure depth-range profiles of various types of seals $[12,13,14]$, which are more easily tagged. These devices were later applied to whales $[14,15]$. Other 
tags were developed specifically for whale depth-range profiles [16] and in many of these $[6,14,17]$, depth measurements were part of a multi-sensor instrument package.

Whales have been detected and tracked passively on seismic arrays and other sonars since the end of World War II. Passive tracking of whales has been used mainly in migration studies and localization of whales during seismic surveys or naval exercises whose horizontal accuracy requirements are low (tens to hundreds of meters) compared to that of behavioral and kinematic studies. Sperm whales, which provide regular and frequent vocalizations, have been tracked passively in recent studies (e.g. [18, 19, 20, 21, 7, 22, 23]). Humpback whales have also been detected and tracked passively $[9,24]$, although with less fidelity due to the irregular frequency of their vocalizations. In addition to passive sonar, active sonar has been used for whale detection and tracking at ranges up to $2 \mathrm{~km}$. These systems have been used primarily for whale-avoidance. Recent work has focused on the detection of whales prior to naval tactical sonar testing $[25,26]$.

In parallel with the development of tags to measure the animal's depth, the advent of digital recording techniques has allowed development of acoustic recording tags for investigating the vocalizations of whales and other marine mammals and the ambient noise around them $[6,14,17]$. To place these acoustic measurements in a behavioral and geographical context, other sensors have been included to facilitate tracking of the animal. For example, the tag developed by Madsen et al. [17] records acoustics, time and depth. The Bioacoustic Probe developed by Burgess et al.[14] records acoustics, depth, temperature and acceleration (for pitch and roll). In addition, the Bioacoustic Probe has been calibrated for flow noise across the transducer allowing estimates of whale speed [13]. These speed estimates provide an independent assessment of whale speed from surface observations allowing kinematic studies of whale movement not attainable by visual sightings alone $[27,15]$. The DTAG used in the development of the acoustic tracking system presented here records acoustics, depth, temperature, acceleration (for pitch and roll), and magnetic heading [6] . DTAGs have not been calibrated for flow noise as the Bioacoustic Probes have been (DTAGs high pass filter their acoustic data, potentially filtering out the frequency bands most likely to 
contain flow information), however the addition of heading allows derivation of $3 \mathrm{D}$ whale tracks not attainable by other tags.

The different methods of tracking described above are appropriate for different applications. For example, long-range, seasonal migrations can be captured by visual sighting and satellite tagging, while VHF tagging is suitable for regional tracking over distances of tens to hundreds of kilometers. When these methods are used to provide a horizontal constraint for depth-time recorders, much can be learned about whale diving and surfacing behavior [11]. In addition, passive positioning can provide 3D tracking with an accuracy of 10 's of meters. Although passive systems are ideal in that they are minimally invasive, passive systems require regularly vocalizing animals. Unlike the other methods, submerged, high-update rate tracking methods provide a means to assess behavior on the scale of the animal's movement. For example, feeding humpback whales commonly blow a vertical curtain of bubbles, known as a bubble net, to corral a school of prey [28]. The size of bubble net curtains, whale speed variation while submerged and the geometry of these characteristic behaviors cannot be captured by surface sightings and can only be measured passively when the animal is actively vocalizing. Tracking of tagged animals through a long-baseline system, such as the one presented here, provides a means to make quantitative estimates of these events.

Traditionally, DTAG measurements of depth and heading are merged with visual sightings to provide a dead-reckoned whale track. Either a constant whale speed is assumed after an initial visual sighting when the tag is applied, or a series of constant whale speeds are derived for intervals between visual sightings throughout the duration that the tag deployment [6]. The assumptions inherent in dead-reckoning produce precise, smooth tracks with a fix-to-fix consistency that is sufficient to surmise the general behavior (e.g. surfacing, diving, bubble net feeding, etc.) of the animal without having necessarily accurately located the animal in a geographic reference frame. Dead-reckoned tracks have been used to visualize the character of whale behavior below the surface [4] in studies involving humpback whale vocalizations $[6,3]$, their feeding habits, and the propensity of ship strikes due 
to shallow feeding depths $[1,2,4]$.

Because dead-reckoned tracks generated from DTAG data are created from an assumed whale speed, they suffer from poor accuracy, provide little information about true whale speed and can induce distortions to the true whale track. Without an independent measure of the whale's position, dead-reckoned tracks are of limited use in studies assessing the kinematics of whale movements or for study of the geometry of characteristic behaviors.

To measure the whale's geo-referenced position and speed, an acoustic positioning system has been developed for the tracking of tagged whales. This long-baseline (LBL) system measures the whale's position at a nominal $1 \mathrm{~Hz}$ update rate providing the ability to track whale movements with sufficient fidelity to assess feeding behavior geometry, whale kinematics and to place the whale in a geo-referenced context. Below we describe a general overview of the system and provide a detailed description of the high frequency acoustic sources involved. We then present DTAG acoustic data processing techniques and approaches to whale positioning as applied to a humpback whale that was tagged and tracked for 80 minutes with the system on July 21st, 2007. Results from this track are discussed, including a comparison of acoustically derived tracks with traditional dead-reckoned tracks and calculation of the speed and geometry of characteristic humpback whale bubble net feeding events. 


\section{CHAPTER 2}

\section{WHALE TRACKING SYSTEM DESCRIPTION}

DTAGs are attached to the whale using a carbon fiber pole from a small rigid hull inflatable boat (RHIB) [6]. The tags are secured to the whale with suction cups and remain on the whale until released by a preset trigger after a specified duration or until they lose suction by other means. While attached, DTAGs provide up to 16 hours of two-channel acoustic recording at $96 \mathrm{kHz}$. Additional instruments aboard the DTAG record temperature, pressure (for depth), three-axis acceleration and three-axis magnetometer data. (Acceleration and magnetometer data are used to resolve whale orientation and heading.) While a tag is affixed to a whale, the animal is followed by three small-boats to monitor the whale's surface behavior and to facilitate recovery of the DTAG when it releases.

The LBL tracking system presented here is deployed to track the whale after the tag is applied and the whale is submerged. The system consists of three acoustic sources, one deployed from each of the three small-boats (Figure 2-1). A simple, hand-deployable, low-power, high frequency, acoustic source was designed for this system. For brevity, the term "pinger" will be used throughout this paper to refer to these sources. Each pinger combines a small microprocessor, secure digital (SD) data card logger, Global Positioning System (GPS) receiver, GPS antenna and power amplifier into a small water-proof case. The system transmits the acoustic signal via a small radially omni-directional transducer lowered approximately $2 \mathrm{~m}$ beneath the surface. A photograph of a unit is shown in Figure 2-2, and a system diagram is shown in Figure 2-3.

The whale-tracking pingers operate in a synchronized fashion utilizing the GPS 1-pulseper-second (PPS) signal as a trigger, thereby sending acoustic pulses at known times and from known locations. The pulses are sent at 25 to $31 \mathrm{kHz}$ (a detailed description of the 
pulse generation is described later), above the estimated frequency threshold of humpback hearing [29]. The pulses are recorded on the DTAG with whale vocalizations and other ambient noise, and by timing the receipt of these pulses on the DTAG, one-way travel times are measured. Ranges from each source to whale are calculated using a nominal sound speed. Finally a range-only least squares solution, constrained by the DTAG-measured depth, provides the 3D location of the whale. Details regarding these data processing steps are provided in Section III below.

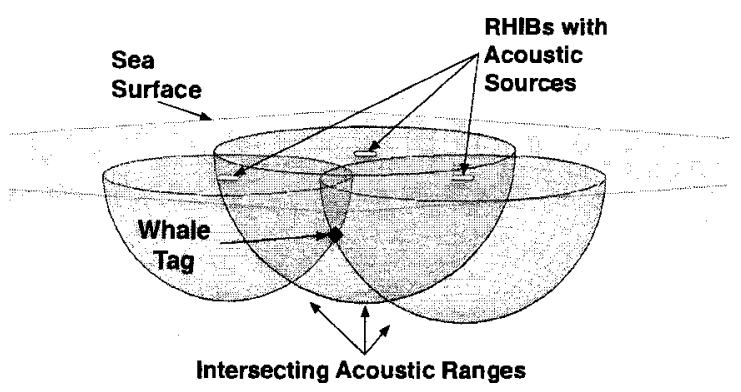

Figure 2-1: Small-boats (typically rigid hull inflatable boats, (RHIB)) follow at several hundred meters from the tagged animal. GPS-positioned acoustic sources are deployed from each RHIB. The acoustic sources are recorded on the whale tag, from which one-way travel times and ranges are measured.

The microprocessor utilized in the pinger was the BASIC Stamp BS2px24. The enabling feature of this processor for this application are the "polling" commands, which provide the functionality of interrupts for a non-multitasking processor, and the "FREQOUT" command which provides a pulse-width-modulated $5 \mathrm{~V}$ signal at a prescribed frequency. These commands allow monitoring of a trigger pin and, on receipt of a trigger signal, generation of an acoustic pulse with very low jitter. Because the Stamp microprocessor has only 16 Kbytes of internal non-volatile memory for program and data storage, an add-on SD card 


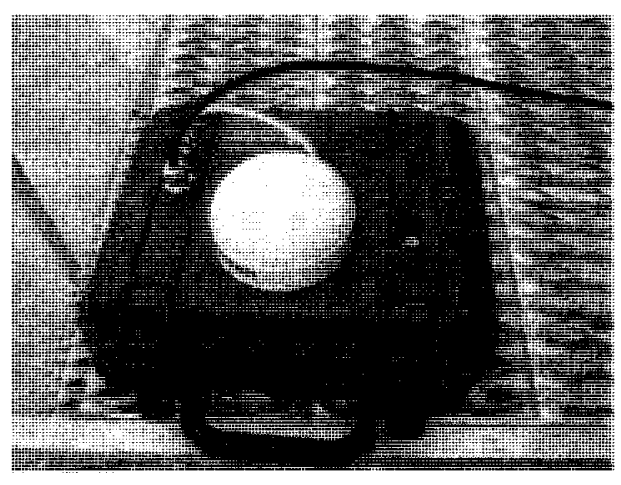

Figure 2-2: Pinger electronics package with surface-mounted GPS, on/off switch and LED indicator light. The case measures $0.235 \mathrm{~m} \mathrm{x} 0.181 \mathrm{~m} \mathrm{x}$ $0.146 \mathrm{~m}$

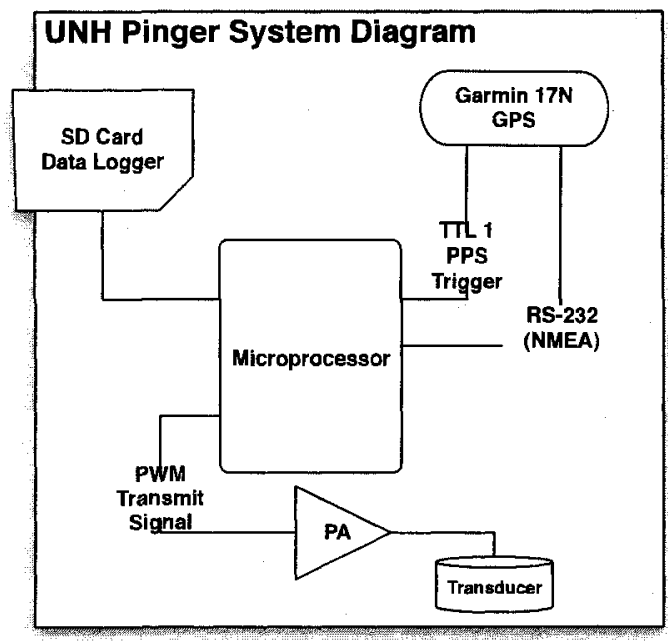

Figure 2-3: Conceptual drawing of the pinger. A Garmin ${ }^{T M}$ GPS provides pinger triggering and positioning. Positions are logged to an SD data card. Pulse width modulated acoustic transmit puises are generated by the control processor, which are amplified and impedance matched to the transducer at the center operating frequency. 
data logger was required for logging GPS positions. Positioning and time-keeping were provided for the pinger by a Garmin ${ }^{T M} 17 \mathrm{~N}$ marine grade GPS. The GPS provides National Marine Electronics Association (NMEA) strings via serial link for position and time at 1 Hz. The GPS also provides a TTL 1-PPS signal synchronized to the UTC second that was used to trigger the pinger for initiation of a pulse. The generated transmit signal (described later) is sent to a power amplifier to drive the transducer.

Power consumption of the system is dominated by the GPS and microprocessor peaking at approximately $1 \mathrm{~W}$ combined. The pulse length, amplitude and duty cycle of the acoustic signal are such that the impact of the amplifier on power consumption is negligible. Therefore a $12 \mathrm{~V}, 2.8 \mathrm{Ah}$ battery can provide an operational lifetime of more than $24 \mathrm{~h}$.

\subsection{System Operation}

Figure 2-4 provides a flow diagram of software operation. When activated, the control processor initializes, and then waits in low-power mode for a trigger signal from the GPS. When a trigger signal is received, an acoustic pulse is sent. Several hundred milliseconds after receipt of the trigger signal, the NMEA position and time string are received from the GPS and subsequently parsed. The GPS data is logged to the onboard SD card and the GPS time is used as a seed in the algorithm to generate the next pulse sequence. These sequence indices are stored and the system returns to low power mode until the next 1-PPS trigger is received.

Measurements were made to assess the latency and jitter of the pinger's acoustic pulses. 1-PPS trigger signals, measured from the GPS receiver of each pinger, were found to vary less than 200 ns. In addition, the delay from receipt of the GPS trigger to generation of the acoustic pulse was measured to be $492 \mu$ s on average with a jitter of $3 \mu$ s (jitter is defined here as one standard deviation of the delay). This trigger-to-transmit delay was accounted for in calculation of one-way travel times. The combined jitter from the 1-PPS trigger signal and that of the microprocessor-generated acoustic pulse contributes just $4.8 \mathrm{~mm}$ of range error (assuming a $1,500 \mathrm{~ms}^{-1}$ ), which was considered a negligible source of error. 


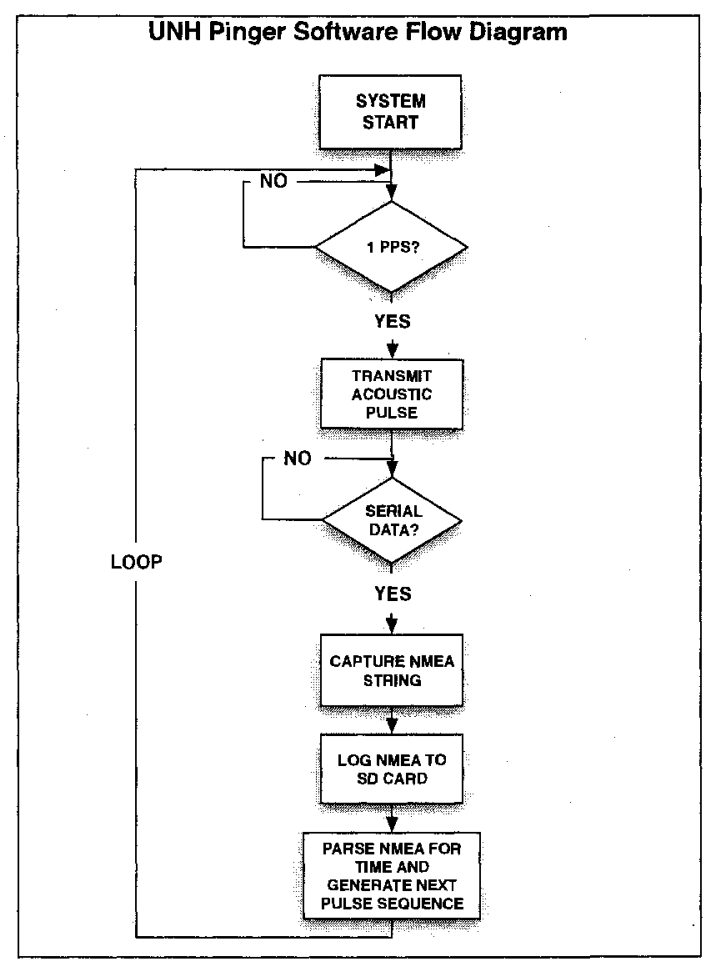

Figure 2-4: Flow diagram of the pinger control processor operation.

In each pulse the pinger sends a train of seven gated, continuous wave (CW) sub-pulses having frequencies of 25 to $31 \mathrm{kHz}$ in $1 \mathrm{kHz}$ steps. The sub-pulses are transmitted for 1 ms each. Each sub-pulse, therefore, has a nominal bandwidth of $1 \mathrm{kHz}$. A complete train of seven sub-pulses provides $7 \mathrm{kHz}$ total bandwidth corresponding to a range measurement resolution of $0.2 \mathrm{~m}$,

In addition, because the DTAG contains only a relative internal time reference, it was desirable to encode UTC time in some manner into each acoustic pulse. This time encoding was used during a DTAG timing calibration step at the end of each deployment. Encoding of UTC time into the pinger pulse was achieved by permuting the seven sub-pulses. Limitations in the operating memory space of the Stamp processor allowed for only simple encoding of the UTC second into each pulse and then only at a resolution of 10 seconds. Therefore, in each of the six 10-second intervals of a UTC minute, a separate permutation was sent. 


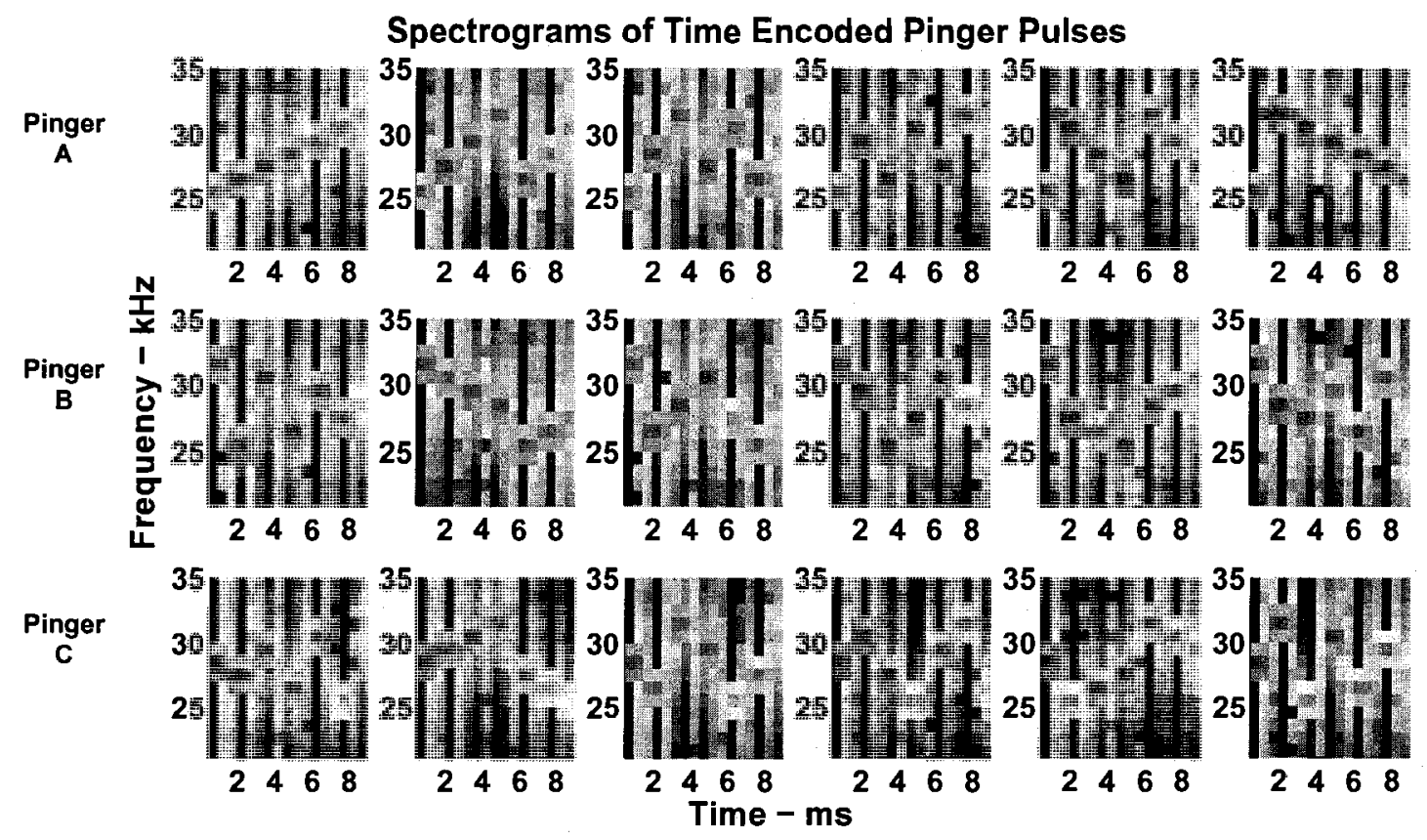

Figure 2-5: Spectrograms of all 18 pinger permutations are shown. Each column of three signals are sent from each of the three pingers in one ten second interval. For example, Pingers $\mathrm{A}, \mathrm{B}$ and $\mathrm{C}$ send the first column of three signals, respectively, for seconds 00-09 of every UTC minute. The three signals in the second column are sent from seconds 10-19 of every UTC minute, and so on.

Since each of these must be unique (to distinguish one pinger from another) a total of 18 pulse types were required for the three pingers.

Non-volatile memory in the microprocessor was insufficient to store the six frequency permutations for each pinger. Therefore, an algorithm was developed to generate the six pulse types from a single unique reference permutation. In the algorithm, a simple ruleset adjusts the order of this reference permutation based on the current UTC second, as reported by the GPS. For seconds 0-9 the reference permutation itself is sent. For seconds 10-19 every other frequency of the reference permutation is sent, returning to the skipped values when the end of the reference permutation was reached. For example, if the reference 
permutation was $[25,26,27,28,29,30,31]$, the permutation sent from 10-19 seconds was generated by initially skipping every other frequency giving $[25,27,29,31,26,28,30]$. For seconds 20-29, the train of sub-pulses was generated by skipping two entries in the reference permutation - e.g $[25,28,31,27,30,26,29]$. Pings for seconds $30-39,40-49$ and $50-59$ were generated by skipping 3,4 and 5 entries respectively. Spectrograms of all 18 pulses are shown in Figure 2-5.

Reference permutations were chosen such that, when comparing any two of the 18 pulses types, no more than three frequencies would match in location either by permutation or rotation of their locations in the signal. This requirement results in a $10 \mathrm{~dB}$ difference between the autocorrelation of each pulse and its correlation with any of the other pulses. While other spread-spectrum techniques are more theoretically supported to encode and detect signals of this type [30], the method used here was simple, and therefore, feasible with the limited capability of the pinger's microprocessor. 


\section{CHAPTER 3}

\section{DATA PROCESSING}

Before processing the DTAG acoustic data several preliminary steps were required. These included generating a temporal reference frame, verifying the DTAG sample rate and generating a matched filter bank. The matched filter bank was then applied to the acoustic data for ping detection. These detections were cleaned, converted to ranges and finally used in a weighted least squares solution for whale position.

\subsection{Generating a Temporal Reference Frame}

Time associated with each DTAG measurement is derived from an activation time and the specified sample rate. The DTAG's internal clock is set manually at a resolution of 1 s when the unit is armed prior to deployment and the activation time (tag-in-water) is recorded internally when the tag is deployed on a whale. Because of the coarseness of the DTAG time reference and the potential variability of the sample rate from tag to tag, it was necessary to generate a high-resolution DTAG temporal reference frame with a UTC time base. The reference frame was established through a timing calibration routine at the end of each deployment after the tag released from the whale.

To conduct the timing calibration when recovering the DTAG, it was held underwater at a fixed, known distance $(20 \mathrm{~cm})$ from a pinger transducer for $1-2$ minutes. The tag recorded the change in pulse types resulting from several 10-second transitions during this time. The date, hour and minute of this calibration step were manually recorded, while the second and fraction of a second were established by detecting a 10-second pulse-type transition measured during the calibration, and correcting the detection time associated 
with this transition for the trigger-to-transmit delay and the delay due to the propagation of the pulse from the pinger to the tag. This propagation delay was calculated from an estimate of the distance between the pinger and tag and a CTD-measured sound speed near the surface. In this way, a sample index in the DTAG acoustic record was correlated with a UTC date and time with a 1-sigma predicted uncertainty of $20 \mu \mathrm{s}$. This uncertainty value is the result of calculation of the propagation of variance due to the uncertainty in the pinger transmit time $(3 \mu \mathrm{s})$, the uncertainty in the propagation distance length $(0.025 \mathrm{~m})$, the uncertainty in the measured sound speed $\left(1 \mathrm{~ms}^{-1}\right)$ and the uncertainty of the detection algorithm $(10 \mu \mathrm{s})$. From the DTAG acoustic sample index correlated with this established time-stamp and a measured DTAG sample rate, a time vector could be calculated for any given segment of data.

\subsection{Verifying the DTAG Sample Rate and Sample Rate Stability}

The method used for generation of the time standard for DTAG data assumes a known DTAG sample rate. To measure the DTAG sample rate and sample rate stability, a controlled experiment was performed, in which each DTAG was attached to a pinger for an extended period with the pinger in operation. Pinger detection times were extracted from the resulting DTAG-recorded acoustic data using an assumed nominal sample rate (initially 96,000.0 Hz). Although a small amount of jitter exists in the interval from ping-to-ping, GPS triggering of the pinger ensures the ping-to-ping duration does not grow or shrink on average. Therefore the slope of a plot of the fractional second of the detection time, i.e. detection time modulo the ping rate $(1 \mathrm{~Hz})$, provides a measure of the difference between the actual sample rate and the assumed sample rate. In the upper plot of Figure 3-1, the fractional second of the receive time for each ping is plotted for the two hour test. The slope of the plot indicates deviation between the assumed sample rate from the correct value, as a sample rate that is too slow produces a growing positive offset, while a sample rate that is too fast produces a growing negative offset. The assumed sample rate was adjusted until the linear portion of the plot was most nearly horizontal (zero slope), as shown. For exam- 
ple, the sample rate for one tag was found to be $96,013.860 \mathrm{~Hz}$ (by comparison, two other tags were found to have sample rates of $96,013.453 \mathrm{~Hz}$ and $96,013.509$, respectively). This sample rate was used for all subsequent calculations for that tag.
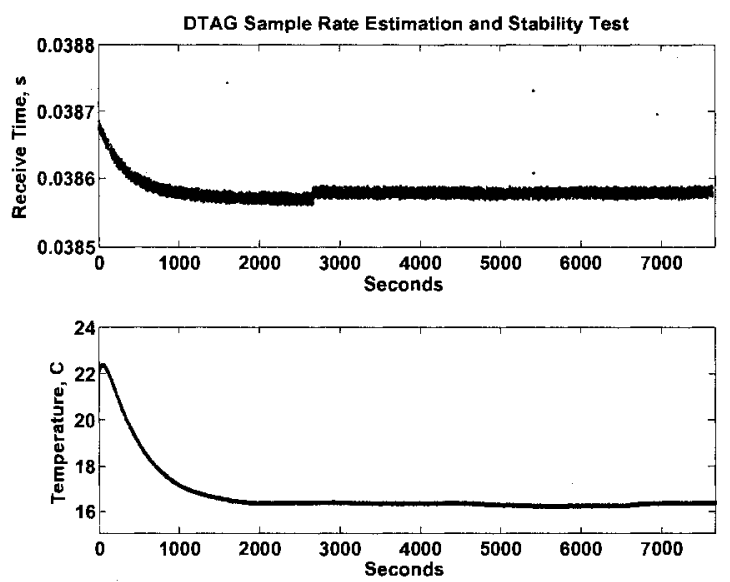

Figure 3-1: To measure the DTAG sample rate and determine the need to characterize it as a function of temperature, a controlled measurement was performed by measuring the pinger at a short, known distance. Receive time after transmission is plotted in the upper plot using an assumed sample rate. The true sample rate was found by adjusting the assumed sample rate until the latter portion of the plot ( $>3000$ seconds) is most nearly horizontal, as shown. Comparison of the receive time and temperature plot (below) shows the tag coming to thermal equilibrium. The tag's oscillator varies here just $0.1 \mathrm{ppm}$ over a $6^{\text {circ }}$ temperature change.

The controlled experiment also allowed verification of sample rate stability over changes in temperature. The effect of temperature on the DTAG sample rate can be seen when comparing the upper plot of ping receive time and lower plot of DTAG temperature in Figure 3-1. A change in detection time of $0.1 \mathrm{~ms}$ is seen over the first 1,000 seconds of operation ( 0.1 parts per million) as the tag comes to thermal equilibrium. The change in temperature over this duration is $6^{\circ} \mathrm{C}$. This variation in sample rate is far smaller than the 
crystal oscillator manufacturer's specification of $+/-30 \mathrm{ppm}$ from $-10-60^{\circ} \mathrm{C}[31]$. In this case, the temperature range fortunately falls near the "zero point" of the AT-cut crystal where the variation in frequency of the oscillator with temperature is very small [32]. In addition, the flat portion of the frequency drift curve extends below the nominal $9^{\circ} \mathrm{C}$ temperature recorded on the DTAG during deployment giving confidence that little variation in sample rate exists between the value measured during the test and an actual deployment. Indeed the data itself exhibits no bias that might be indicative of significant sample rate variation.

Careful inspection of the receive time in Figure 3-1 will also reveal a few false detections and a step function in the received time at approximately the 2,700 second point. The false detections are an artifact of the detection algorithm and may be ignored for the purposes of this plot. The step function results from a processing irregularity in the control processor of the pinger. Steps of $10 \mu$ s similar to this one occur in a random way with no net change in the trigger-to-transmission delay. While the cause of this irregularity is unknown, its size was never seen to vary significantly. Therefore, the irregularity is expected to impart no significant error to the positioning ( $10 \mu$ s equates to just $15 \mathrm{~mm}$ of range error).

\subsection{Processing the Acoustic Data}

Acoustic data from the DTAG were band-pass filtered from $24 \mathrm{kHz}$ to $32 \mathrm{kHz}$ and baseband demodulated. A UTC time vector was then created from the start time established in the timing calibration step and DTAG sample rate. The acoustic data were segmented by UTC second and the correct matched filters for each 10 second interval and pinger were applied. The matched filter library was generated for the 18 pulse types by measuring each pulse in the University of New Hampshire's acoustic test tank facility. RMS amplitudes and durations of each sub-pulse within each permutation were measured. These values were then used to generate analytic models of each complete pulse at the desired sample rate.

Because the time-encoded pulses generated by the pingers contain the same base frequencies, portions of any two pulse-trains will correlate with each other above the noise floor. Therefore, any given matched filter in the library will generate at least a small cor- 
relation peak when correlated with any other pinger signal. The peak, however, is largest when the correct filter (i.e. the one that actually matches the signal) is applied.

Correlation output above a preset threshold was identified as the beginning of a candidate window of time for each detection. This detection threshold was set dynamically at 20 times the root mean square noise measured in the previous second. The multiplicative factor of 20 was determined empirically to ensure true detections could clearly be identified within an abundance of false detections and to ensure that manual cleaning of the resulting data in a subsequent step was not prohibitively time consuming. False detections were common, due in part to frequent whale surfacings, which appear as broadband noise in the tag data, and an EK-60 fisheries sonar operating at a center frequency of $38 \mathrm{kHz}$ from a nearby support ship. These influences caused the change in noise levels to overwhelm the detector's ability to adaptively adjust the detection threshold.

Each detection candidate window provides a duration within which an individual pinger detection is identified. The detection candidate window length was set to at 0.75 times the pulse length (about $7.5 \mathrm{~ms}$ ). This length provides a balance between prevention of multiple detections in close succession, resulting from a window that is too long, and unwanted multiple detections from a single ping, resulting from a window that is too short. Within a detection candidate window, the individual matched filter output of the three pingers having the largest value identifies which pinger was detected. A detection time can then be recorded as the UTC time corresponding to the index of that peak.

\subsection{Calculation of Pinger-to-DTAG Ranges}

Ranges were calculated using the one-way travel time and a nominal speed of sound in seawater. Each ping is sent on the UTC second after a fixed trigger-to-transmit delay. For any given detection, the one-way travel time is the detection time in seconds modulo 1 , minus this trigger-to-transmit delay. To simplify processing, the effects of varying sound speed with depth (i.e. both refraction and sound speed along the travel path) were ignored and a single nominal value of $1,500 \mathrm{~ms}^{-1}$ was used. The use of a constant, $1,500 \mathrm{~ms}^{-1}$ sound 
speed provides a gross estimation of the whale's range. However the range bias due to this type of error is slowly varying with time, preserving the consistency of the measurement from fix-to-fix. In addition, because the bias is applied to all range measurements, a favorable geometry, in which the whale is roughly equal distant and between the three sources, removes the effect of this bias in the final position estimation.

All false detections and multipath arrivals were cleaned from the data set manually. Although others have used multipath arrivals for localization of whales using matched field processing $[24,19]$ the intent here was to keep the model and processing as simple and computationally tractable as possible. The criterion for cleaning involved selecting measurements that together provide a visible, continuous, trace of data points through an otherwise random cloud of false detections, omitting multipath traces when they could be clearly identified. An example of raw data, with points retained after cleaning circled, can be found in Figure 3-2. A plot of all the resulting acoustically-measured ranges from each pinger to the DTAG can be found in Figure 3-3.

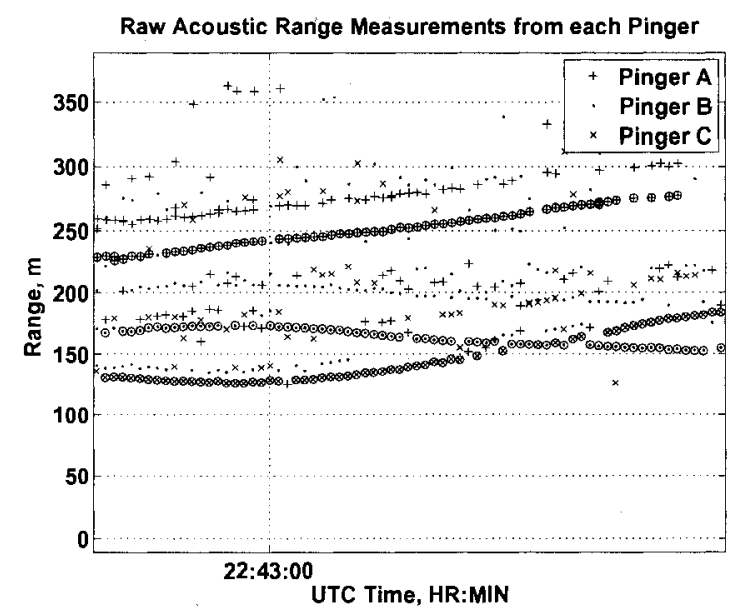

Figure 3-2: A portion of the raw range measurements from each pinger to the whale are shown. A nominal $1,500 \mathrm{~ms}^{-1}$ sound speed was used to calculate ranges. Circled points indicate those that were retained after a manual cleaning step. Multipath arrivals are frequently evident as parallel lines of data beyond the first arrivals. 


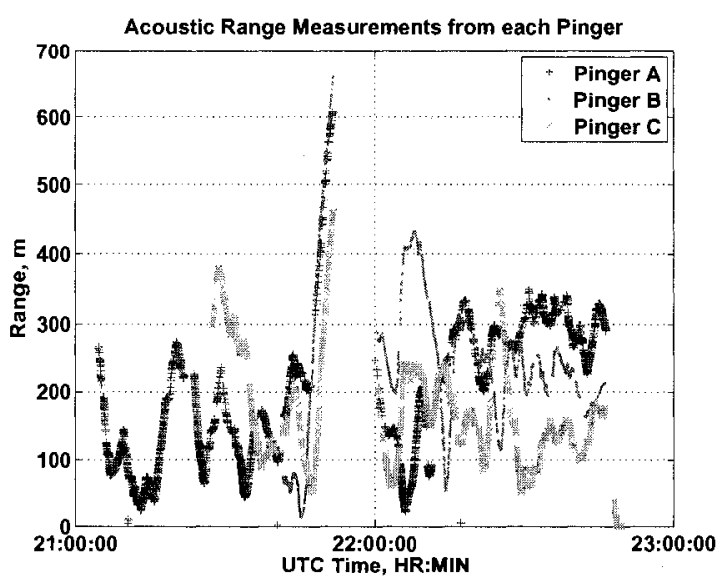

Figure 3-3: Individual range measurements from each pinger to the whale are shown. A nominal 1,500 $\mathrm{ms}^{-1}$ sound speed was used to calculate ranges. The apparent discontinuity prior to 22:00 resulted from a repositioning of the pingers when the whale left the immediate feeding area to join other whales several kilometers away at a speed faster than the small-boats could maintain with the pingers deployed.

\subsection{Least Squares Positioning}

When ranges were measured from all three pingers in a single one-second interval, a nonlinear least squares solution for whale position was calculated. In this calculation, the vertical position was forced to the DTAG measured depth, as the geometry of the RHIB boats with respect to the whale poorly resolves position in the vertical direction due to the fact that the horizontal ranges are 2 to 6 times larger than the maximum whale depth.

In an effort to maximize the number of whale position estimates, solutions were also calculated when only two of the three pingers were detected. Two intersecting spheres of range produce a circle of possible whale locations which may be further reduced to a semi-circle of possible locations below the water line. A separate measurement of depth (in this case measured by the DTAG's onboard pressure sensor) reduces the number of possible solutions to two locations which must be resolved by comparison with other solutions and 
visual observations from whale surfacings.

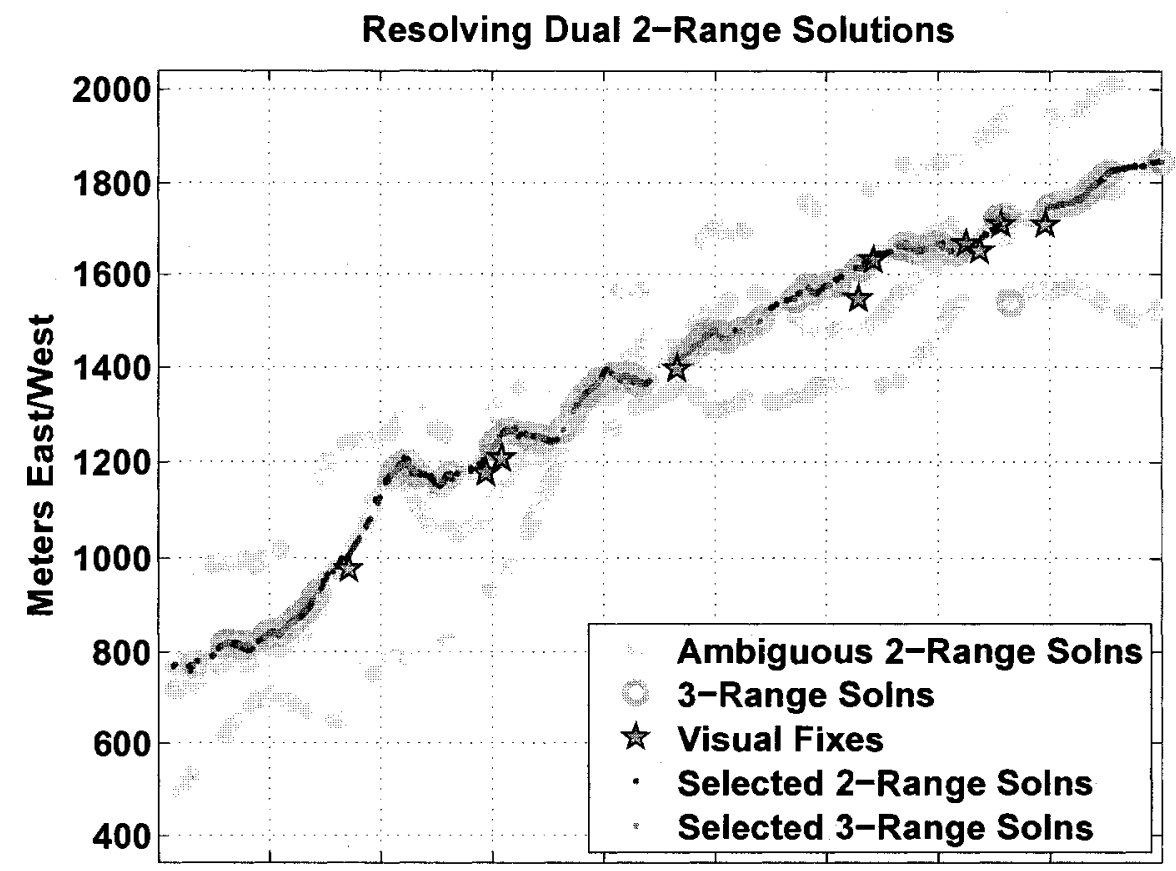

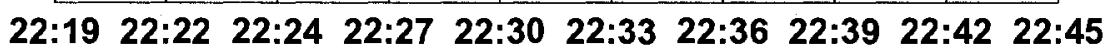
Deployment Time, HR:MM

Figure 3-4: To disambiguate two-range solution pairs, all fix position information is plotted on a single time-series plot such as the subset of data shown here. For each two-range solution pair, the solution's east/west coordinate is plotted vs time. In addition east/west coordinates from 3-range solutions and visual fixes of the whale while at the surface are plotted. The combined data set allows one to select which of the ambiguous two-range solution pairs is correct using the unambiguous 3 -range solutions and the visual fixes as a guide. Outlier 3-range solutions were also omitted. The process is repeated for north/south coordinates and the intersection of the measurement times from each provide the final two-range solution set. All positions are plotted here as meters from the mean value.

To resolve the two ambiguous dual-range solutions, as much information about the 
whale's true position was plotted versus time and the most likely correct position was chosen by manual comparison. Figure 3-4 illustrates the technique, in which east/west coordinates (in meters from the mean) are plotted vs. time for all two-range solutions. Visual fixes and three-range solutions are plotted on top of the two-range solutions and are used as a guide to disambiguate the two-range solution pairs. Outlier 3-range solutions were also omitted in the process. The identical method is used for north/south coordinates. The intersection of measurement times that correspond to the east/west coordinates and the north/south coordinates are used to generate final data set.

To provide a measure of uncertainty in the least-squares solution, an estimate of the uncertainty in each pinger-to-whale range measurement was propagated through the leastsquares calculation. Because of the bandwidth of the acoustic signal and sample rate of the DTAG, uncertainty in the measurement of one-way travel time was small $(<1 \mathrm{~m})$ when compared to the uncertainty in the GPS-measured position of the pinger, and therefore, the GPS uncertainty was considered alone for a gross approximation. The GPS position $95 \%$ confidence, radial uncertainty is $15 \mathrm{~m}$ [33]. 


\section{CHAPTER 4}

\section{EXAMPLE WHALE TRACK}

On July $21^{\text {st }}$, 2007, a humpback whale, named "Geometry," was tagged just prior to the end of the day's tagging efforts at approximately 21:00 UTC (5:00 PM EST). Geometry was bubble net feeding with "Venom," "Coral," "Etch-a-sketch" and a fifth unidentified animal at the northern tip of Stellwagen Bank (Figure 4-1a). Bubble net feeding is unique to humpback whales, in which a circular curtain of bubbles is blown to corral a school of prey [28]. The whales subsequently engulf the prey as they are trapped within the curtain and the surface. Geometry was tracked with the LBL system for approximately 80 minutes.

Geometry was initially tagged by a team operating from a small-boat a few kilometers from the two other small-boats and support ship in the science party. After the tag was applied, the team deployed pinger A, which shows up first in the time-range record shown in Figure 3-3. The remainder of the science party relocated to the area and the two other small-boats deployed pingers $\mathrm{B}$ and $\mathrm{C}$ shortly thereafter. After bubble net feeding for several minutes in the same locale, Geometry and his feeding pod transited at a rapid rate to another school of prey approximately one kilometer away. The small-boats were unable to keep pace with the whales while the pingers were deployed, therefore the pingers were recovered, the boats repositioned and the pingers redeployed. This break in track is evident at 21:50 in Figures 3-3 and 4-1b. Geometry was tracked by the three small-boats through several dives and bubble net feeding events before the tag released at 22:46.

The entirety of the acoustically derived whale track for Geometry is shown in Figure 4-1b in which the whale track data is plotted over gray-scale shaded bathymetry. Seafloor depths beneath the track range from approximately 35 to $65 \mathrm{~m}$. For clarity, shadows of the track

have been draped over the bathymetry and the vertical dimension has been exaggerated by 


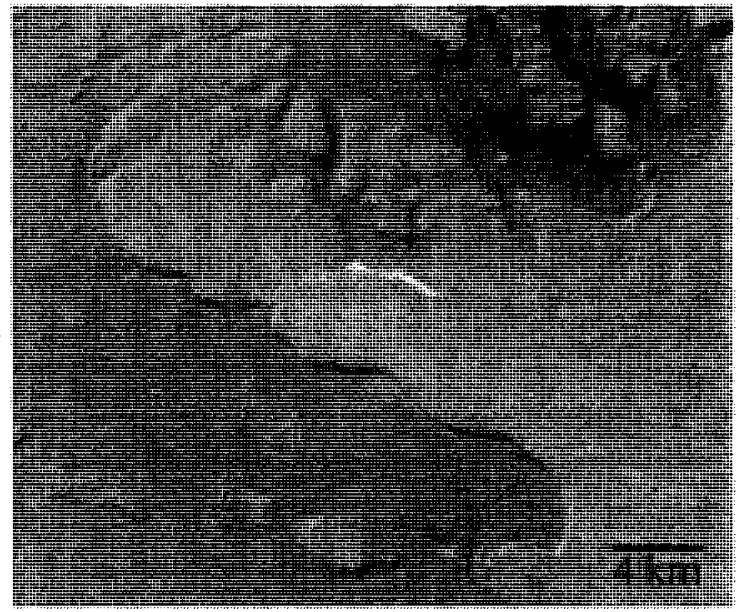

(a)

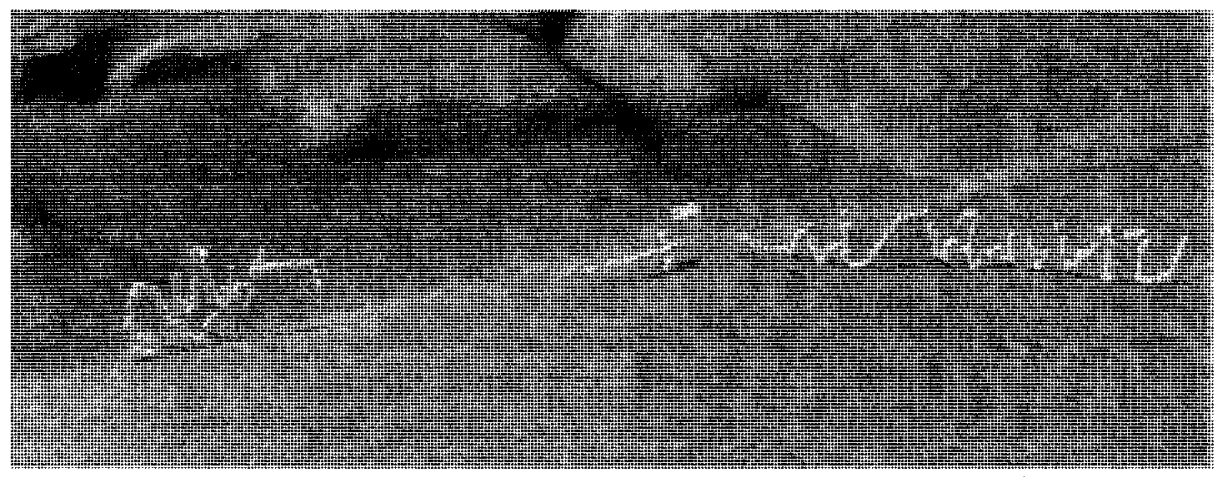

(b)

Figure 4-1: Plan and perspective views of the acoustically-derived track for the whale "Geometry" shown over local bathymetric data. Shadows of the track data points have been draped on the bathymetry in black for clarity. The perspective view is looking to the North-Northwest from atop Stellwagen Bank. Vertical exaggeration is 10x. The track duration is approximately 80 minutes and the general path of the whale is from left to right. 
a factor of ten. The image is looking to the North-Northwest and Geometry's movement is from left to right coving some 4 kilometers from start to finish.

\section{$\underline{4.1}$ Comparison with Dead-Reckoned Tracks}

The independent, acoustically derived, whale track provides an opportunity to validate and compare the traditionally generated dead-reckoned tracking methods. Figure 4-3 compares portions of the acoustically derived data (left), with a dead-reckoned track created assuming

a constant velocity of $1 \mathrm{~ms}^{-1}$ (right). The left plot of figure 4.1 shows a subset of the acoustically derived whale track from 22:00 to 22:45 in which the whale was moving from east to west. The left hand plots of each of Figures $4-3 \mathrm{a}, 4-3 \mathrm{~b}$ and $4-3 \mathrm{c}$ focuses on a small portion of that subset. The right side of Figures 4.1, 4-3a, 4-3b and 4-3c show the corresponding constant-velocity, dead-reckoned whale track for the identical timeframes. Points in all plots are measured from the same arbitrary point (the mean of the Eastings and Northings of the acoustically measured data).

Figure 4.1 shows a small-scale view of a portion of the acoustic and dead-reckoned whale track data so that differences in the general trend of the data can be seen. Horizontal distance actually covered by the whale is much larger than the distance illustrated in the dead-reckoned whale track (2200 $\mathrm{m}$ vs $1700 \mathrm{~m}$ respectively). Considering only starting and ending points, the mean overall horizontal speed for the acoustic track data of 0.83 $\mathrm{ms}^{-1}$, while that of the dead-reckoned track is just $0.43 \mathrm{~ms}^{-1}$ indicating that the assumed instanteous whale speed of $1 \mathrm{~ms}^{-1}$ may be off by as much as a factor of two. Moreover, comparison of the N/S and $\mathrm{E} / \mathrm{W}$ axis of each plot shows that, because the dead-reckoned track was fixed with only a single visual sighting at the beginning (approximately 1.6 hours before), errors in whale speed and direction have accumulated quickly. In this case, the deadreckoned positions have drifted by $800 \mathrm{~m}$ in the north/south direction and $1400 \mathrm{~m}$ in the east/west direction. The general direction of whale movement in each plot is also different - with less movement to the south shown in the dead-reckoned track. This difference in direction may indicate a heading bias in the whale tag data. 


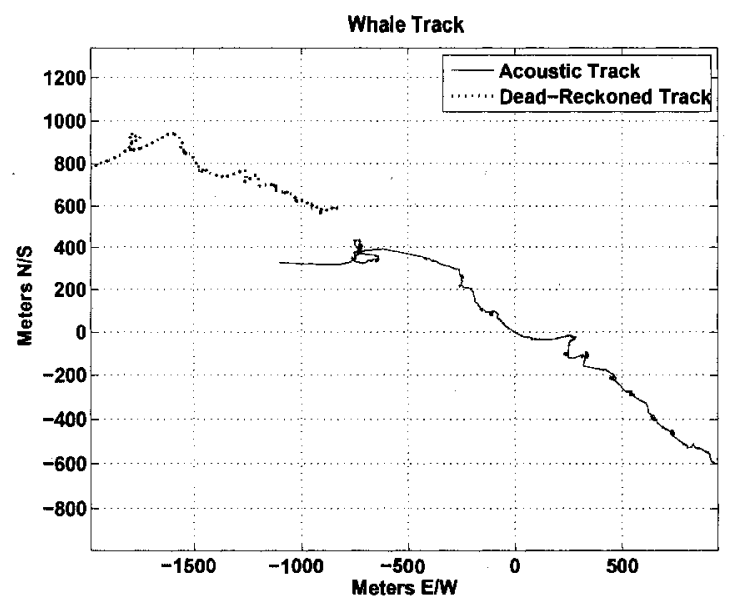

Figure 4-2: A portion acoustically measured whale track is shown with dead-reckoned whale track for the same time. Points in both tracks are plotted from the mean of the acoustic data. The dead-reckoned whale track is generated from an initial visual fix approximately 1.6 hours prior to the data shown here, and an assumed $1 \mathrm{~ms}^{-1}$ whale speed. Errors in the dead-reckoned track over this time frame accumulate quickly resulting in a track offset by nearly $2 \mathrm{~km}$.

The plots in Figure 4-3 focus on a smaller portions of the same data set. Figure 4-3a contains three bubble net feeding events, which are evident as circular loops in these planview plots. The characteristic bubble net loops are clearly evident in both the acousticallyderived and dead-reckoned whale tracks.

Figure 4-3b provides a closer comparison during a single bubble net feeding event. In the dead-reckoned track data, two loops are clearly evident as the whale travels a helical path, rotating twice while moving vertically through the water column. However, close inspection of the acoustic data in Figure 4-3b reveals that most of the second loop is not recorded. The loss of fix data during the maneuver of a second loop during ascent occurs in several of the tracked bubble net feeding events. (This is the case for all three events Figure 4-3b, for example.) Inspection of the raw detection data (not shown here) indicates that this loss of track results from a loss of direct path acoustic propagation of the pinger 


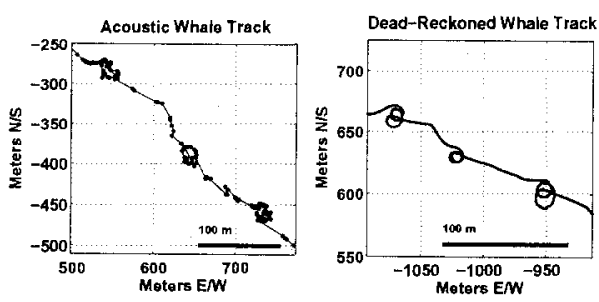

(a)

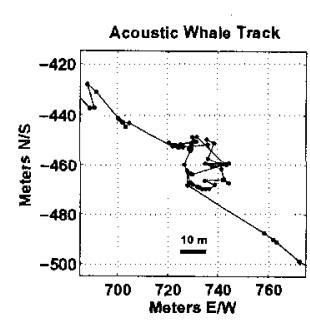

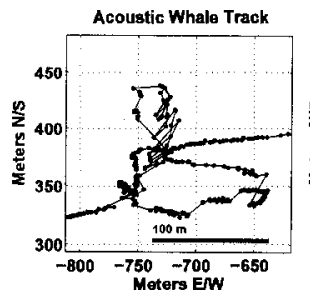

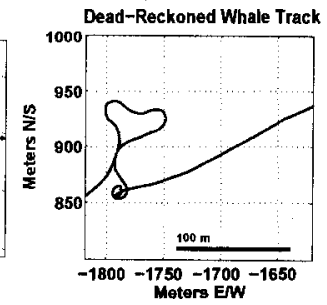

(b)

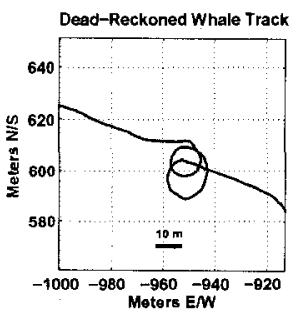

(c)

Figure 4-3: The pairs of plots provide a closer comparison of the acoustically derived and dead-reckoned whale tracks shown in Figure 4.1. The left plot in each subfigure shows acoustically derived whale positions, while the right plot shows dead-reckoned track with an assumed constant velocity for the same time as each of the plots on the left. In each plot the whale enters from the east and departs to the west. All positions are measured relative to the mean Eastings and Northings of the acoustically derived positions.

signals to the tag. This might result from acoustic attenuation of the signals by the bubble net curtain. In future work it may be possible to retain track on the animal through the bubble net curtain, as bottom bounce signals are often still present.

Finally, comparison of the plots in Figure 4-3c show qualitatively different results between acoustic and dead-reckoned whale tracks. In this case the acoustic data is particularly noisy due to the fact that, for a short duration, all three pingers and the whale were in a straight line in the general north/south direction. This poor geometry provides little to no constraint to the position estimates in the east/west direction. The result is a noisy track which bears little resemblance to the dead-reckoned track. 
As shown in these plots, when unconstrained by visual fixes, dead-reckoned tracks poorly locate the whale in a geographic reference frame. None-the-less dead-reckoned tracks can provide a qualitative measure of the whale's movement such that the general behavior of the whale is easily inferred. Acoustically derived whale tracks, on the other hand, georeference the whale, giving a proper dimension to its movements that is unattainable from dead-reckoned tracks. Acoustically derived tracks are not without their own limitations, as acoustic attenuation of the pinger signals may cause a loss of track and poor geometry may produce poor fixes.

Dead-reckoned tracks may be constrained by visual fixes to reduce the positional bias that accumulates over time. Efforts to do so, however, can change the character of the track in ways that may not be desirable. For example, Figure 4-4a shows an acoustically derived whale track for three bubble net feeding events, while Figure 4-4b shows the dead-reckoned track generated from a single constant speed and Figure 4-4c a visual-fix constrained deadreckoned track, all for the same time period. The fix-constrained dead-reckoned track is relatively accurate in absolute position-within several 10s of meters of the acousticallyderived data. However, because the visual fixes cannot capture the dynamics of whale speed at depth, a dead-reckoned track constrained by them tends to "string out" vertical movement. The effect is shown in these bubble net feeding events, in which a vertical helical whale path is strung out horizontally. In this case, the unconstrained dead-reckoned track reproduces the qualitative character of the whale's behavior (if not its exact position) with greater fidelity.

\subsection{Whale Track Measurements}

In addition to geographically constraining dead-reckoned whale tracks, acoustically-derived positions allow estimation of horizontal whale speed during transits and the geometry of common maneuvers. Horizontal whale speed is of particular interest because the depth measurement on the DTAG provides a reasonable estimate of vertical speed leaving the horizontal components constrained only by periodic surface observations. Seven straight- 


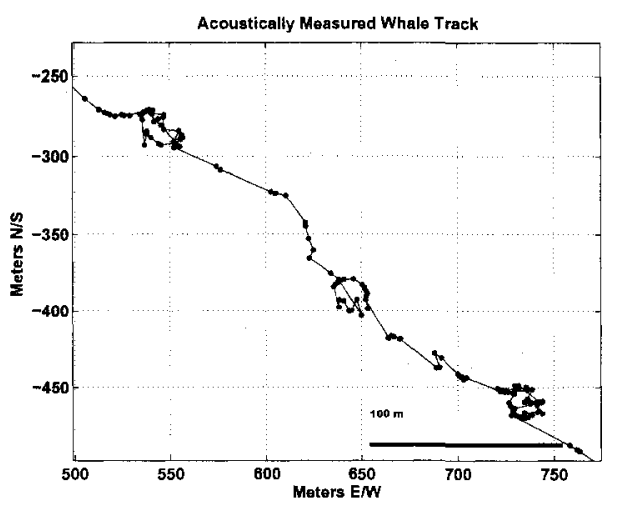

(a)

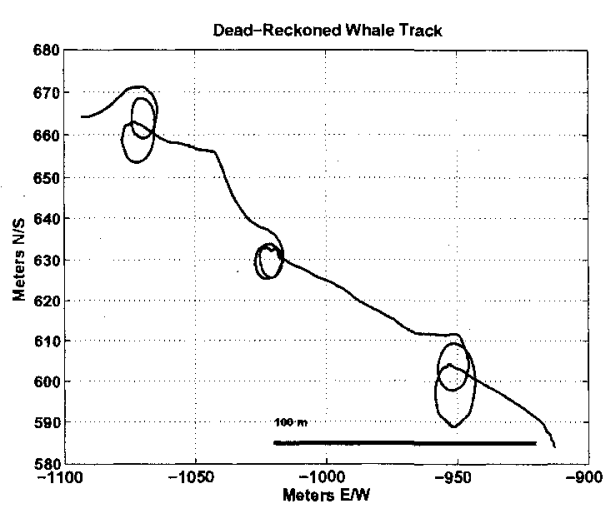

(b)

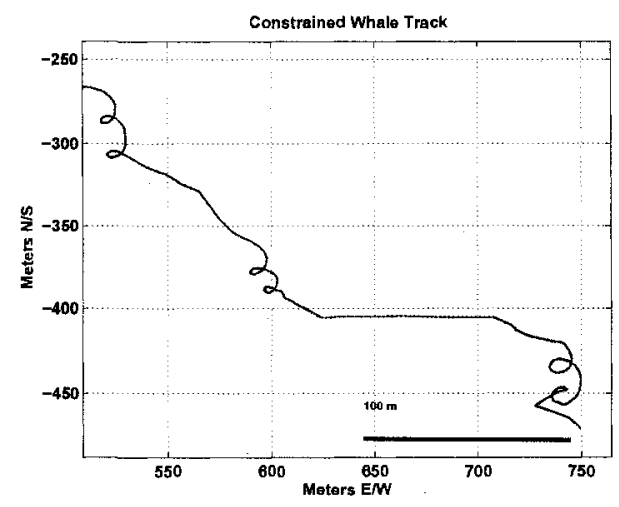

(c)

Figure 4-4: An acoustically-measured whale track is compared with a constant-velocity pseudo-track and a pseudo-track whose velocity is constrained by visual sightings while the whale is at the surface. Although the fix-constrained pseudo-track is more geographically accurate, the surface fixes cannot capture changes in whale speed while at depth, resulting in the "stringing out" horizontally of vertical bubble net feeding events. Comparison with the acoustically-measured positions shows that, in this case, the non-constrained pseudo-track captures the whale behavior with better fidelity, although the absolute positioning is poor. 
line segments were extracted from the acoustically derived positions to estimate horizontal whale transit speed between bubble net feeding events. The events were extracted from a whale track during which the whale was nearly continually feeding-moving from bubble net maneuver to bubble net maneuver. The mean transit speed for each segment was calculated by dividing the distance between the first and last positions by the travel time. This method was preferable to integration of the distance traveled over the path divided by the travel time, as noise in the acoustic measurements results in an overestimation of whale speed. A line was fit to the acoustically measured points and the coefficient of determination, also known as the $R^{2}$ value, for each fit was calculated using

$$
R^{2}=1-\frac{S S_{\text {err }}}{S S_{\text {tot }}}
$$

where $S S_{e r r}=\Sigma\left(y_{i}-f_{i}\right)^{2}$ and $S S_{t o t}=\Sigma\left(y_{i}-\bar{y}\right)^{2}$. Here $y_{i}$ are the measurements, $f_{i}$ is the line-fit data and $\bar{y}$ is the mean of the measurements. The $R^{2}$ value indicates, on a scale of zero to one, a quantitative measure of the amount the whale deviated from a linear path and therefore a measure of the robustness of the speed measurement. For example, segment number 2 (Figure 4-5) has an $R^{2}$ value of 0.98 , giving high confidence to the 4.2 $\mathrm{ms}^{-1}$ value. Similarly, segment number 3 has an $R^{2}$ value of 0.58 , in which case either the positions were of particularly poor quality or the path taken by the whale was not linear. The seven calculated transit speeds are shown in Figure C-1. Local currents are not accounted for in these calculations; currents in this area are typically less than $0.2 \mathrm{~ms}^{-1}$ [34]. The mean travel time for the seven segments was 77 seconds.

This small dataset is insufficient in size and scope to make or confirm generalizations about humpback swimming speeds, yet they are the first measurements of this temporal and spatial resolution. Transit speeds measured here provide comparable results to a recent larger study of migrating humpback whales [35]. In that study, average transit speed for whales migrating between feeding and breeding grounds were measured over 10 hour average time segments. Mean transit speed was reported at $1.1 \mathrm{~ms}^{-1}$ as compared to the average $1.4 \mathrm{~ms}^{-1}$ transit speed calculated here (omitting the $4.2 \mathrm{~ms}^{-1}$ measurement which is probably atypical). The similarity in these measurements is likely due, in part, to the 


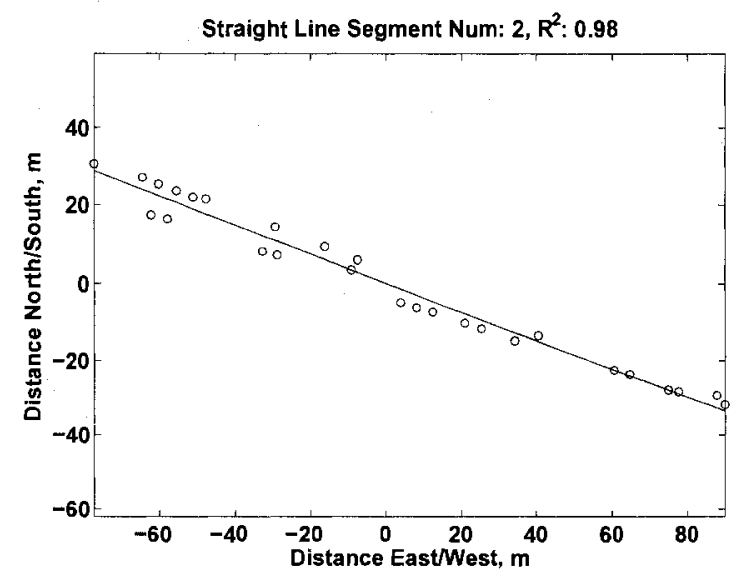

Figure 4-5: Example of a straight-line segment used to estimate transit speeds in Figure C-1.

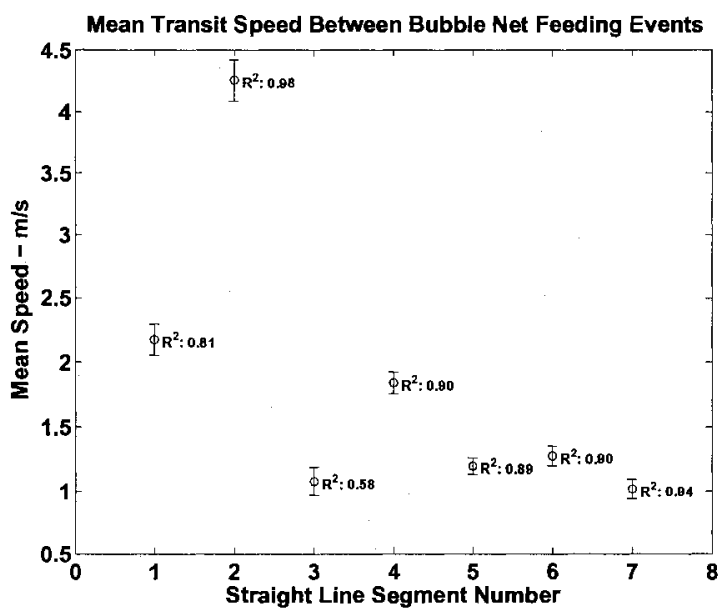

Figure 4-6: Transit speed estimations between bubble net feeding events.

optimal swimming speed of the humpback whale, in which the energy per distance traveled is minimized [36].

The same study of migrating whales reports a maximum observed transit speed of 6.5 $\mathrm{ms}^{-1}$ over just a 12 minute period which may be compared to the maximum observed transit speed here of $4.2 \pm 0.2 \mathrm{~ms}^{-1}$. Transit speeds of this magnitude are uncommon and are likely a sprint for the animal, in which a school of prey or perhaps avoidance of some threat is more advantageous to the whale than the energy saved due to swimming with maximum 
efficiency. As more acoustic track measurements of this type are acquired, a measure of a typical whale's ability to accelerate and maintain high swimming speeds could be developed. These of kinds of observations may provide useful guidance to the development of protocols for commercial shipping to mitigate the potential for ship-strikes, in that they may provide estimates of the likelihood that, when a whale detects imminent danger, it can successfully avoid it.

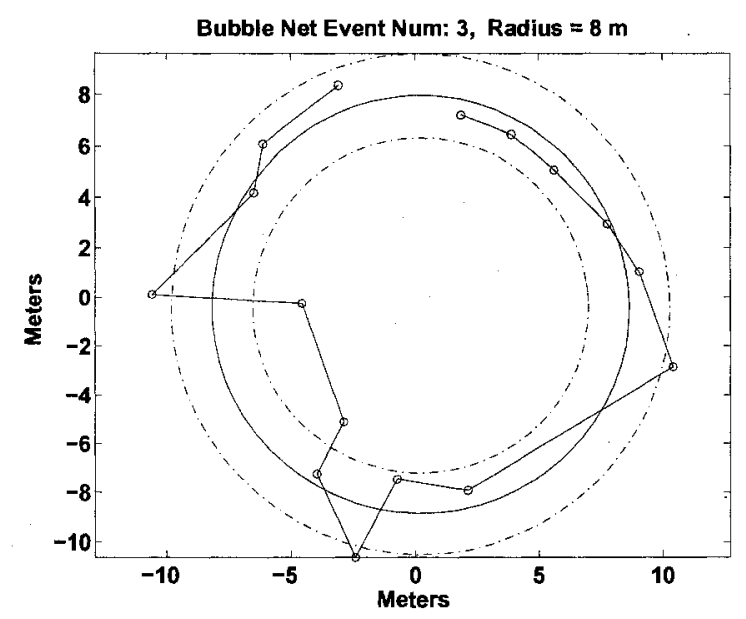

Figure 4-7: Acoustically-measured positions during a bubble net feeding event are plotted, along with a least squares fit of those positions to a circle The one-sigma uncertainty bounds are plotted as dotted lines. Identical methods were used for four other bubble net events whose results are shown in Figure 4-8.

Acoustically-derived positions were analyzed from five bubble net feeding events and a circle has been fit to the data from each under the assumption the whale travels a circular horizontal path during the blowing of bubble net curtains. An example of acoustically derived positions and the resulting fit is shown in Figure D-1. The radii from all five events are shown in Figure 4-8.

It is unknown exactly what factors dictate the size of a bubble net curtain. It has been hypothesized that the size of the net may be adjusted by the blowing whale depending 


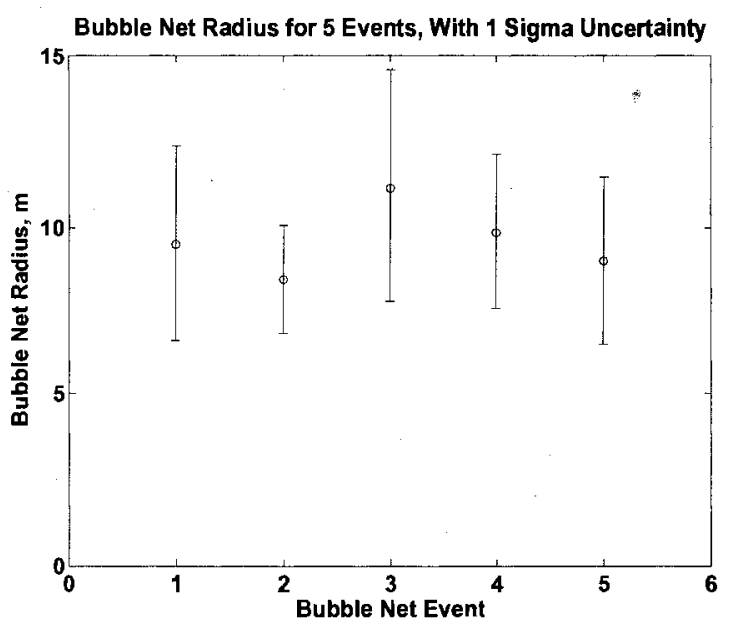

Figure 4-8: Bubble net radius measurements derived from a least-squares fit to acoustically measured positions.

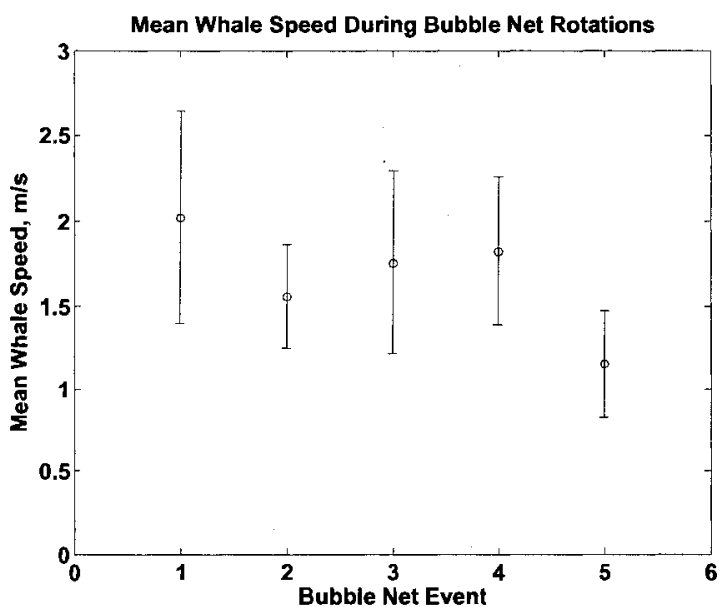

Figure 4-9: Horizontal mean whale speed estimates during whale rotation and ascension while bubble net feeding.

on the number of whales in the foraging pod [28]. In the measurements presented here, four other whales were present. No data was presented in the previous study allowing comparison of bubble net sizes and the number of whales with the measurements presented here. Conceivably, the radius of a bubble net may also be related to the sprint speed of the prey, as a larger bubble net cylinder would be required to engulf a faster-moving school. Or the radius of the bubble net may be only as large as the whale has air to blow, thereby 
providing a clue to the volume of air that may be held by a humpback whale. These questions will remain unresolved until more whales are tracked in different environments and while feeding on different prey.

From the radius of each bubble net event and times associated with the start and stop of rotation, mean horizontal whale speed has been calculated for each using Equation 4.2,

$$
\text { WhaleSpeed }=\frac{2 \pi r \Omega}{t_{\text {end }}-t_{\text {start }}}
$$

where $r$ is the radius fit to the bubble net circle, $\Omega$ is the fraction of the circle the whale traveled, and $t_{\text {end }}$ and $t_{\text {start }}$ are the ending and starting times of the maneuver, respectively. The resulting speeds are shown in Figure 4-9.

These measurements may provide important clues to the way in which whales craft bubble net curtains. Sharpe [28] suggests a strategy used by humpback whales to minimize the formation of gaps in bubble nets as a function of the volume of air blown and the depth of the whale. Larger volumes of air released at a given instant tend to produce larger, leading bubbles. Although the bubbles rise quickly, separating them from other bubbles initially with the potential to cause gaps, the leaders subsequently shed a large effervescence plume which constructs the bubble curtain beneath them more effectively than a smaller initial volume. In addition, when half the cross-sectional area of adjacent bubbles overlap they tend to coalesce into larger bubbles. Sharpe suggests, therefore, that by overlapping bubbles, humpback whales may produce larger leading bubbles to produce a more effective effervescence curtain. Sharpe concedes that it is unknown what volume of air may be held by a humpback and therefore does not consider the speeds at which a whale might move while blowing bubbles to ensure bubble overlap. Speeds of humpback whales during bubble net feeds measured in this study range from $1-2 \mathrm{~ms}^{-1}$. Therefore, given an estimated bubble size at the time of blowing, an estimate of the volume of air blown by the whale may be made. Additional work would be required to make such an estimate.

By providing geo-referenced positions, the acoustic positioning system also allows the study of the potential for the whale's interaction with the sea floor. For example, Hain et 


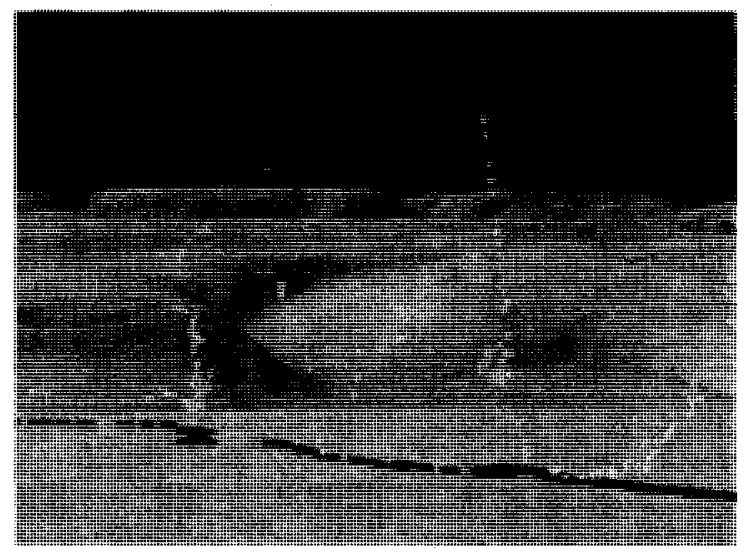

Figure 4-10: Three whale dives during feeding events are shown in this track segment. Shadows of each whale position have been draped on the sea floor for clarity. Estimated whale positions intersect the sea floor for the center and right dives, indicating probable actual contact with the sea floor: The whale's movement is from left to right in this image. Vertical exaggeration is $10 \mathrm{x}$.

al. [5] describe abrasions and scarring common on the lateral, lower, jaw of many humpback whales, apparently caused by contact with the sandy, shell-ridden bottom. Combined with other indirect evidence, they hypothesize that humpback whales may bottom feed on Stellwagen Bank [5]. Figure 4-10 provides a 3D perspective of acoustically measured positions from three dive events between bubble net feeding events shown over bottom bathymetry. Without other evidence it is not clear the whale was bottom feeding, none-the-less, acoustic positioning allows one to demonstrate the whale's position with respect to the sea floor and thereby corroborate theories of how they might interact with it. 


\section{CHAPTER 5}

\section{CONCLUSION}

A system for long-baseline acoustic positioning of tagged humpback whales has been developed with sample updates at $1 \mathrm{~Hz}$ and estimated positioning uncertainty at the 5 m, 1-sigma uncertainty level. The system, consisting of three pingers, may be deployed easily by hand from a small-boat and will operate for the duration of the time the acoustic recording tag is attached to the whale. When the tag's sample rate has been measured prior to deployment, only a short timing reference calibration is required on retrieval of the DTAG.

Stability of the DTAG sample rate is essential for the system to operate for long durations. Typical ocean water temperatures $\left(5-20^{\circ} \mathrm{C}\right)$ serendipitously fall on a plateau of stability for the crystal oscillator driving the DTAG analog-to-digital converter. Were this not the case, the effect could be mitigated by placing the tag in a cold water bath prior to deployment such that they undergo only a small temperature change when deployed.

Results from the system have helped to validate dead-reckoned tracks that are traditionally created from DTAG heading and depth measurements alone. Dead-reckoned tracks that assume a constant whale speed for the duration of the deployment have been shown to generally reproduce the qualitative character of the true whale track, but have poor absolute positioning. On the other hand, dead-reckoned tracks whose speed is derived from visual fixes at the surface constrain absolute positioning, but have been shown to distort characteristic whale movements, such as bubble net feeds, in which the majority of the movement of the whale is vertical. Acoustically derived whale positioning provides geo-referenced positions which better represent the true motion of the whale and from which quantitative measures of that motion may be made. The acoustically derived track may suffer from 
acoustic attenuation from bubbles during bubble net feeding events and occasional poor geometry between the pingers and the whale. These trade-offs lead to a natural blending of the two methods in some optimal way, to be developed in future work.

Transit speeds between bubble net feeding events have been estimated from the acoustically derived positional data. These speeds range from 1 to $4 \mathrm{~ms}^{-1}$ with an average non-sprint speed of $1.4 \mathrm{~ms}^{-1}$. These results are similar to studies of migrating humpback whales. In addition the radii of several bubble net feeding events have been measured from the acoustically measured whale track. Values range from 8 to $11 \mathrm{~m}$ for a foraging pod of five whales. It remains unclear what trade-offs exist in the size of bubble net curtains, however the answer may lie in the number of whales, the speed of prey and the volume of air a humpback may hold. Horizontal whale speeds have also been calculated for the whale during the blowing of bubble nets. These values range from 1 to $2 \mathrm{~ms}^{-1}$. Measurements of whale speeds while blowing bubble net curtains may provide insight to the strategy employed by humpbacks to ensure a gap-free bubble net and to the volume of air that may be held by a humpback. Acoustically derived whale tracks, when combined with bathymetric data, other ancillary tag sensors and corroborating evidence from other studies can provide convincing evidence that humpback whales bottom feed in the Stellwagen Bank National Marine Sanctuary. In future work, assessment of time spent near the bottom may help to assess the propensity for entanglement in bottom fishing gear. 
APPENDICES 


\section{APPENDIX A}

\section{STAMP BS2PX CODE}

The following is the entirety of the code for operation of the pingers described in this the-

sis. Slight modifications were made (commenting and uncommenting lines as appropriate)

for each unit.

, $\$$ STAMP BS2px

, \$PDRT COM1\}

\{\$PBASIC 2.5\}

Val Schmidt, Andy McLeod, Tom Weber

, $\mathrm{CCOM} / \mathrm{JHC}$

May 2007

This code provides smarts FOR the CCOM GPS-triggered pinger.

General Operation:

The system starts in standby-mode, blinking a green LED at . $2 \mathrm{~Hz}$, and

waiting for you to push the start button. On a 5 second button push,

, the system will be placed in PING mode. It will then monitor the GPS_PPS pin,

, and ON receipt DUTPUT a ping. The ping type can be specified in the

' RUNTIME VALUES section (CW AND Chirp are currently available. IF CW is

, selected you can specify a pulse length. The default CW frequency is $30 \mathrm{kHz}$.

, IF Chirp is selected a series of $4 \mathrm{~ms}$ long $\mathrm{CW}$ pulses will be sent at $25-31 \mathrm{kHz}$

, at $1 \mathrm{kHz}$ intervals. The sytem will immedially monitor the GPSSERIAL PIN FOR

, incoming NMEA strings. It will parse the \$GPRMC string, capturing the

, time, lat and lon. These will be written TO EEPROM. If instead on startup,

' the start button is pushed AND held FOR 10 seconds, the green LED will

, flash twice rapidly and the system will dump the contents of the EEPROM

, out the DATA serial port. The EEPROM will not be erased until the pinger

, is placed in PING mode again.

, TO DO:

1 1) Insert code that looks as the "POSVALID" variable of the GPS string

, and only pings/records data when "A" is received. Why? After the GPS is

'started up, if it looses its fix, POSVALID will contain "V", meaning

' the system is in dead-reckon mode. We probably don't want these values

, 2) This is a big one - figure out how to handle more memory. This will

, likely require either another eeprom, or spitting the data out the serial

- port to another system for logging (which could theoretically be done

' wirelessly). For a full day of data, we'd currently need about 10, $8 \mathrm{k}$

- eeproms. Fortunately eeproms come in larger sizes.

3) Need to write a GPS config function that will configure the GPS to a

, known state automatically on startup.

, 4) At the moment, the GPS is powered separately with no switch. We

, should control power to the GPS with the STAMP, and keep it powered down

, until the activation button is pushed. This will probably require a small

, transister, as I don't think you can power the GPS through a pin on the

, stamp.

15) Since the addition of EEPROMs for data storage is not figured out yet,

- two things remain to be coded. DUMPDATA code hasn't been written.

, Bugs:

, 1) Should probably use the BUTTON function for the button, to prevent

spurious nose from triggering the system.

2) At the moment, on startup the system blinks the LED with a 5 second pause in between until the button is pushed long enough for the system

to catch it and activate the pinger. Ideally the 'pause' be a 'sleep'

statement so we can save some power. Unfortuantely with sleep statements

I found erratic behavior I couldn't control. Specifically, sometimes

I found erratic behavior I couldn't control. Specifically, sometimes

one would have to push the button through several sleep durations before

the system would catch the button push and activate.

3) There's no error checking on the pulse length specification. We should 


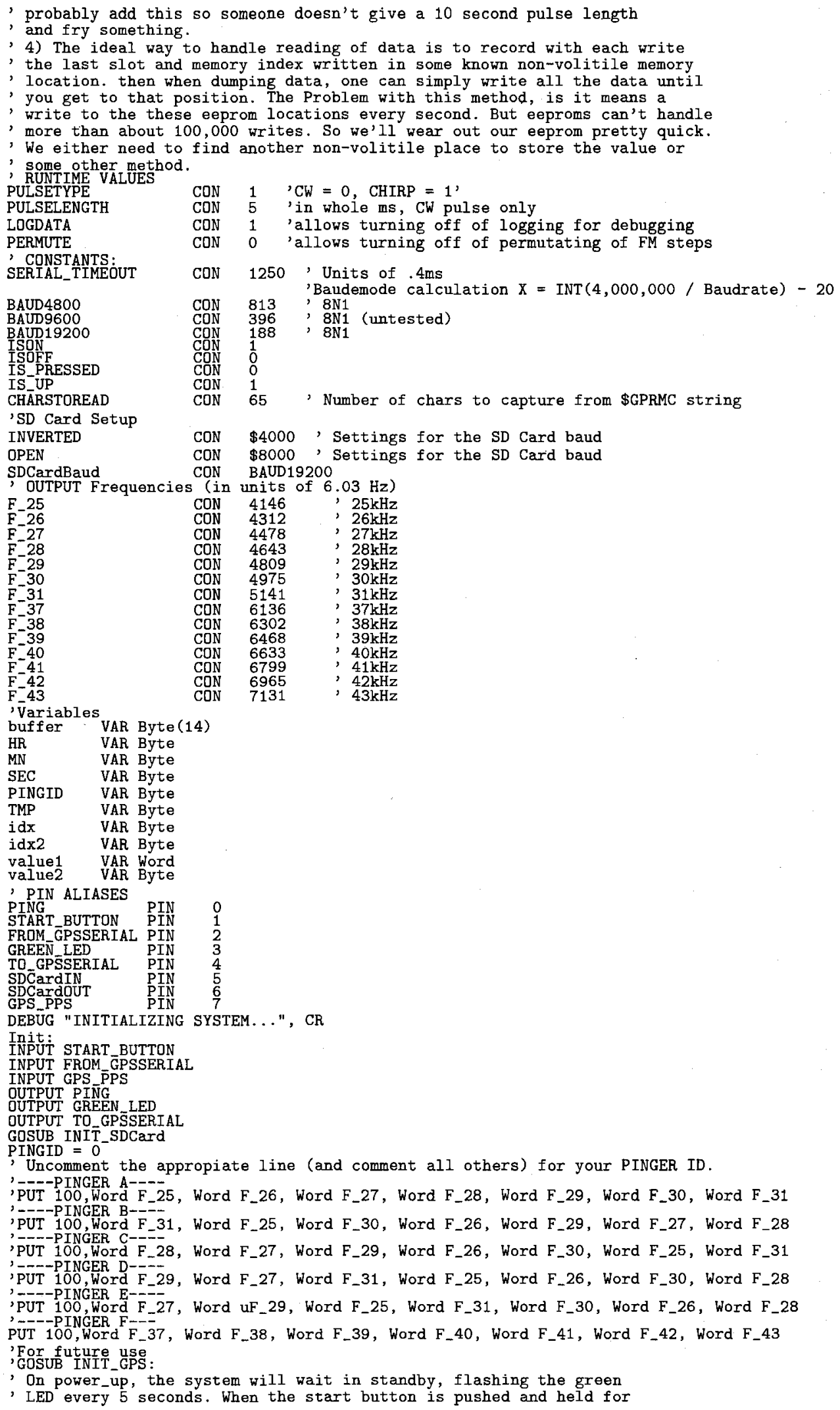




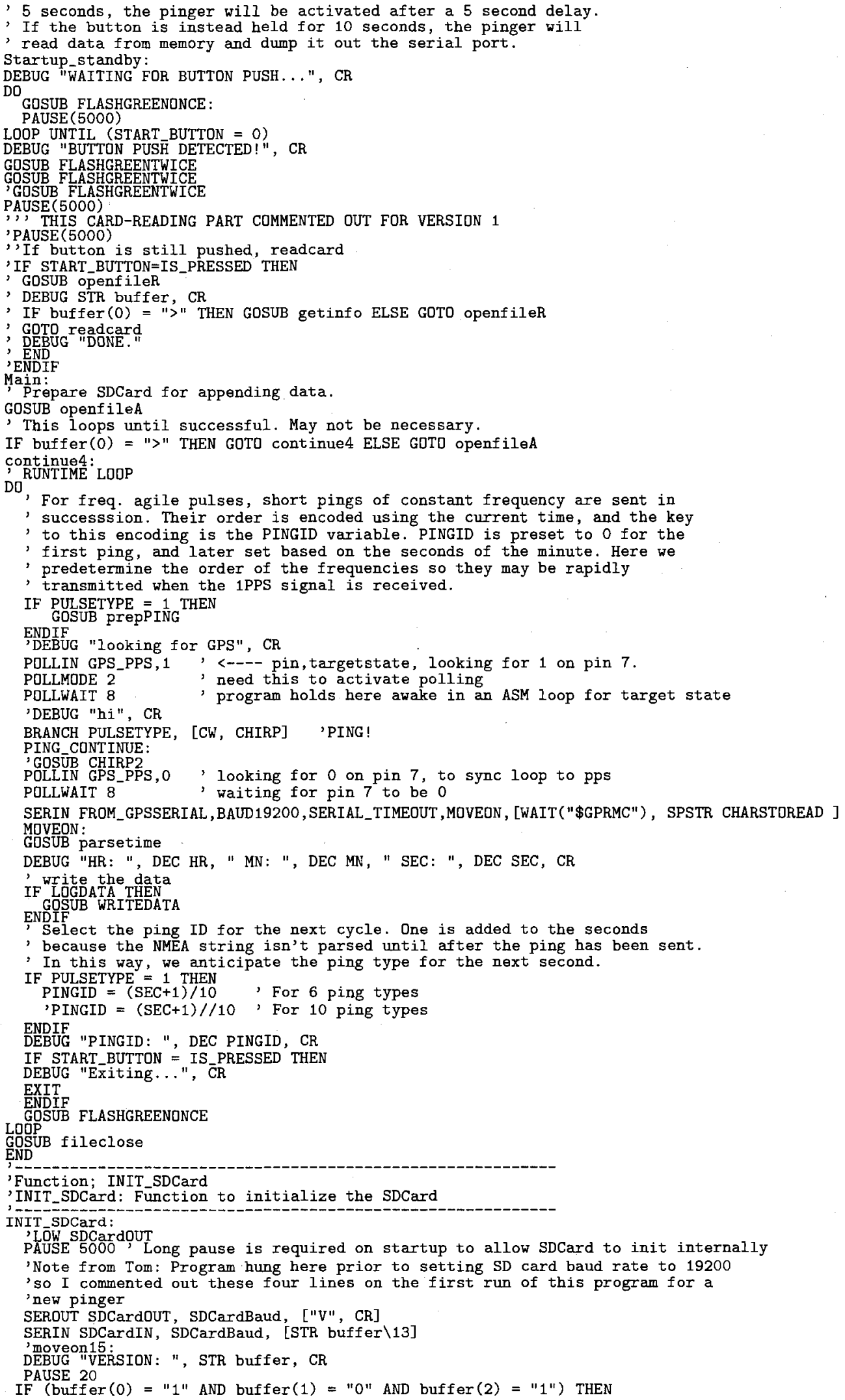




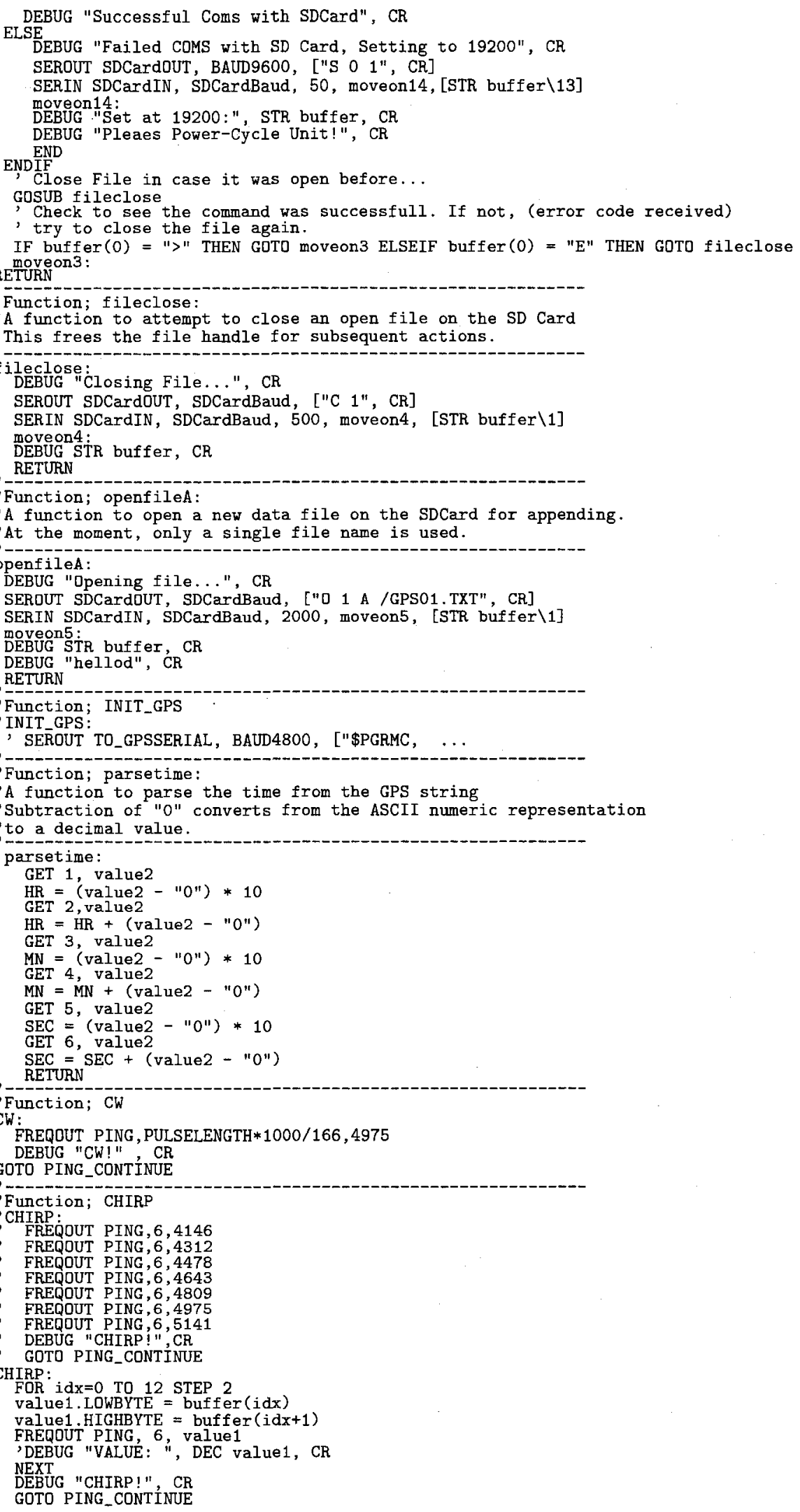




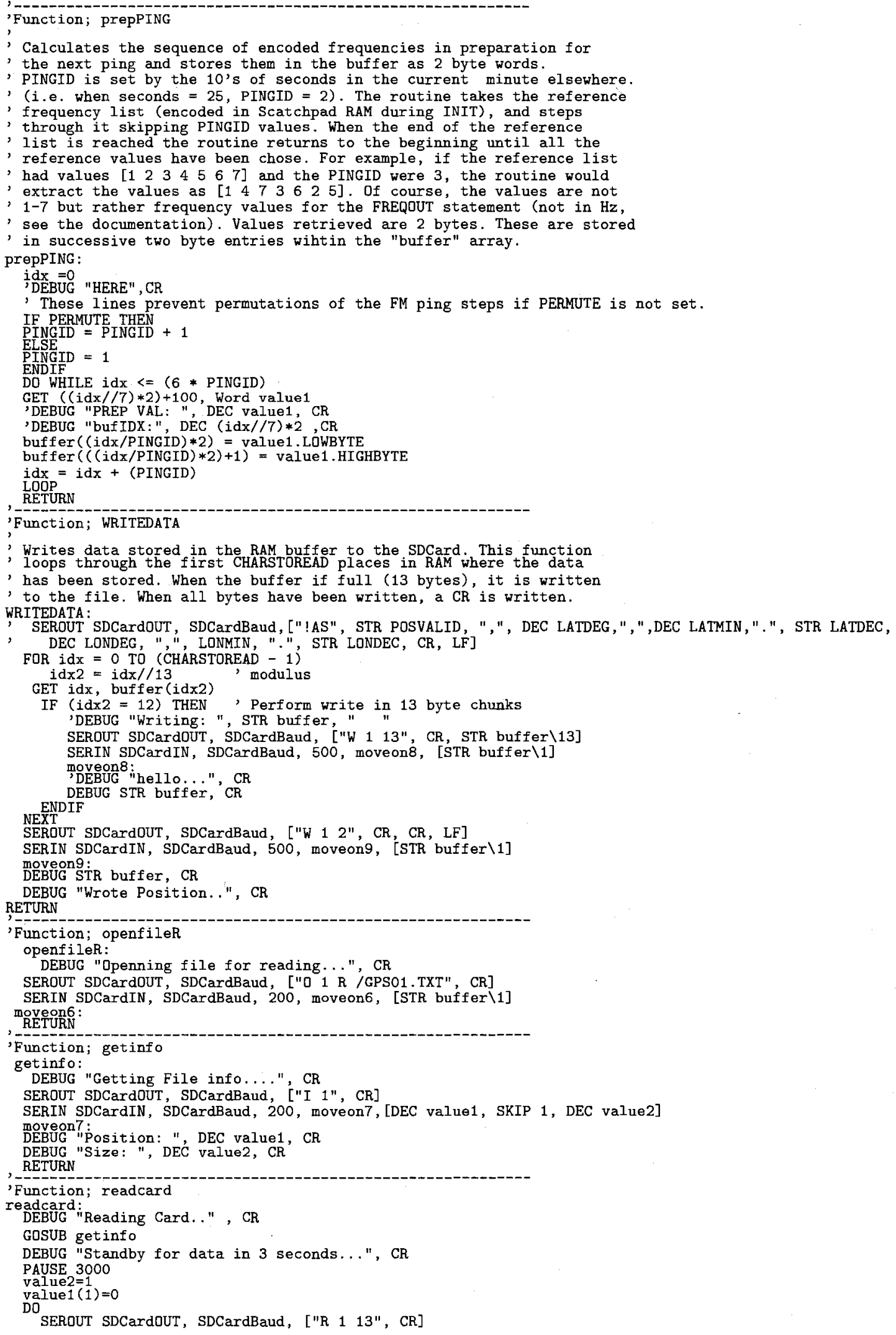




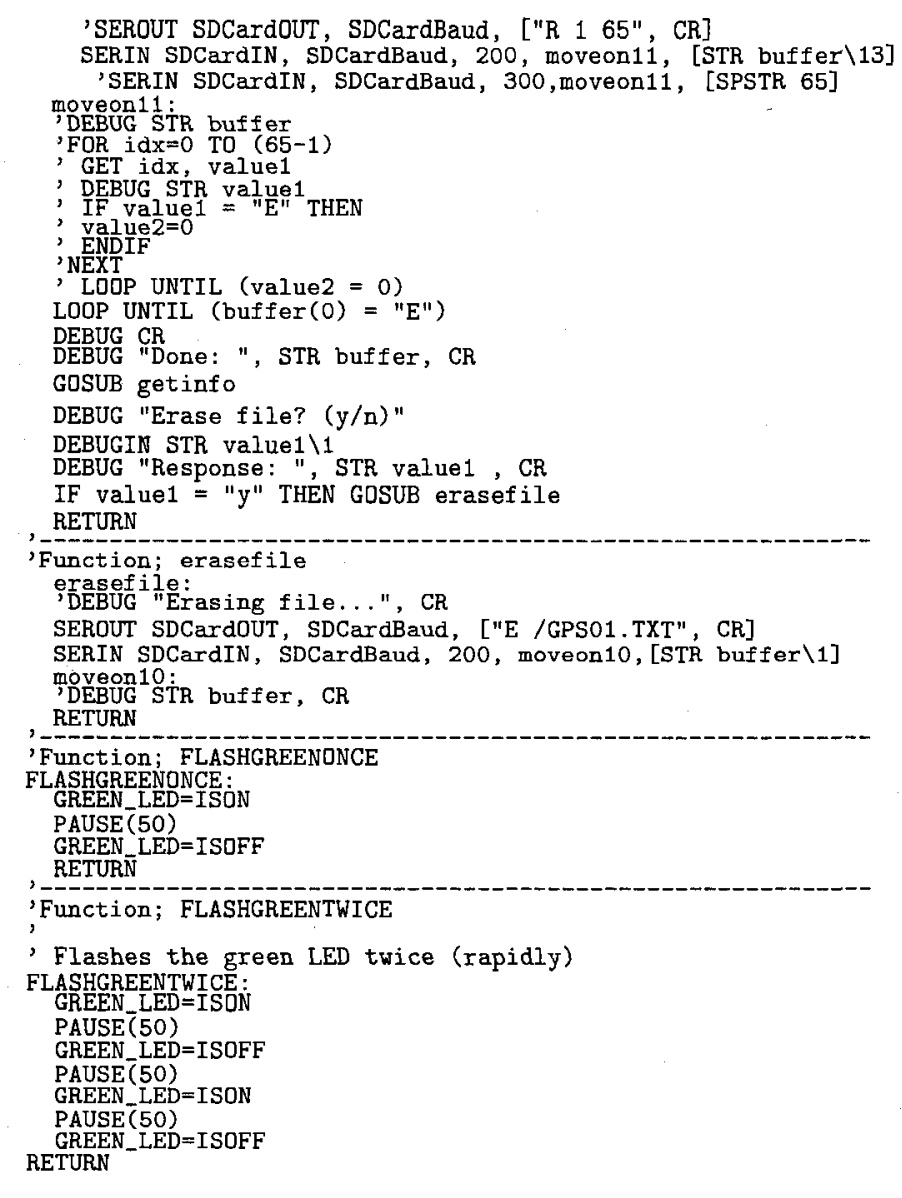




\section{APPENDIX B \\ MATCHED FILTER BANK GENERATION}

To generate the matched filter bank, operation of each pinger was measured in the University of New Hamsphire's acoustic test tank facility. Amplitude of each sub-pulse in the train is a function of both the frequency of the pulse and its relative order in the train. For example the power amplifier was tuned to match the transducer impedance at a nominal $30 \mathrm{kHz}$. Therefore sub-pulse's having frequencies above or below $30 \mathrm{kHz}$ result in smaller amplitude signals. In addition, although a $4400 \mu \mathrm{F}$ capacitor is used to provide a current boost to the power amplifier this current is not maintained throughout transmission resulting in reduced amplitudes for sub-pulses later in the train. An example of a recorded pinger transmission is shown in Figure B-1. The size of the acoustic test tank is not large enough to prevent the simultaneous recording of both direct path and reflected signals. To mitigate the effect of reflected signals, the pinger transducer and hydrophone were placed in close proximity $(; 1 \mathrm{~m})$ such that the received signal was far greater than any reflected signal from the tank walls or surface.

To generate the matched filter bank, acoustic samples of all 18 transmit pulses were recorded and amplitude weighting factors calculated for the sub-pulses in each.

Figure B-3 shows the results of measured amplitude weighting factors for one pinger. These weights $\left(w_{1} \ldots w_{7}\right)$ were used in Equations B.1 - B.7 to generate the subpulses for each matched filter. 


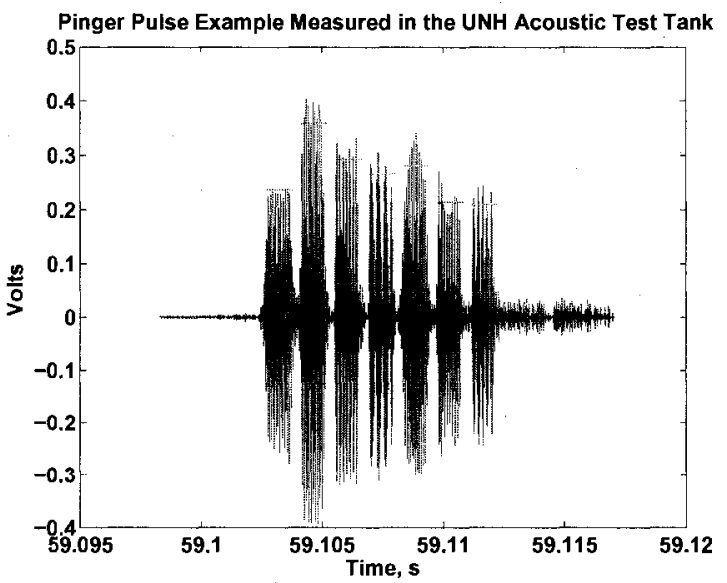

Figure B-1: An example waveform of a pinger transmission recorded in the the University of New Hampshire's acoustic test tank. Relative amplitudes of each sub-pulse are a function of both the frequency of the pulse and its relative position within the larger train.

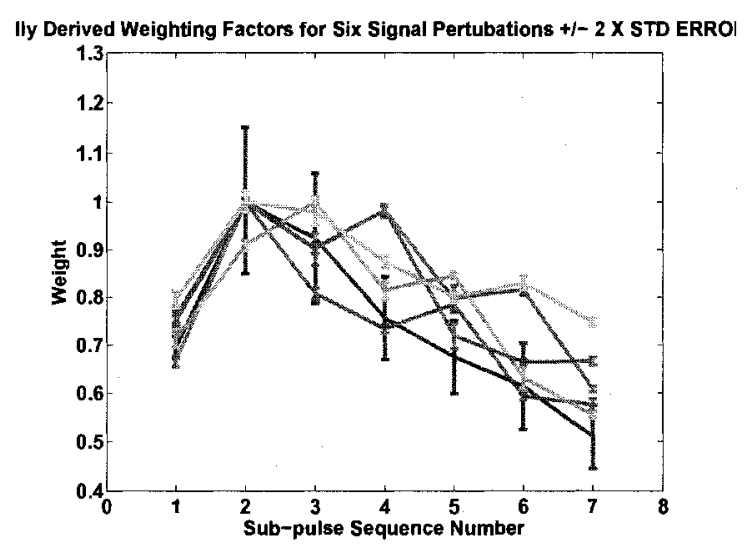

Figure B-2: Mean acoustic sub-pulse amplitude weighting factors measured from a full minute of recorded transmissions from one pinger. Since the sub-pulse permutation changes every 10 seconds, 10 amplitude values are averaged to determine each weight. 


$$
\begin{aligned}
& \text { subpulse } 1=w_{1} \sin \left(2 \pi f_{1} t\right) \\
& \text { subpulse } 2=w_{2} \sin \left(2 \pi f_{2} t\right) \\
& \text { subpulse } 3=w_{3} \sin \left(3 \pi f_{3} t\right) \\
& \text { subpulse } 4=w_{4} \sin \left(4 \pi f_{4} t\right) \\
& \text { subpulse } 5=w_{5} \sin \left(5 \pi f_{5} t\right) \\
& \text { subpulse } 6=w_{6} \sin \left(6 \pi f_{6} t\right) \\
& \text { subpulse } 7=w_{7} \sin \left(7 \pi f_{7} t\right)
\end{aligned}
$$

The subpulses were then concatenated with short durations of zeros inserted between each to produce the final matched filter pulse train shown in Figure B-3 below.
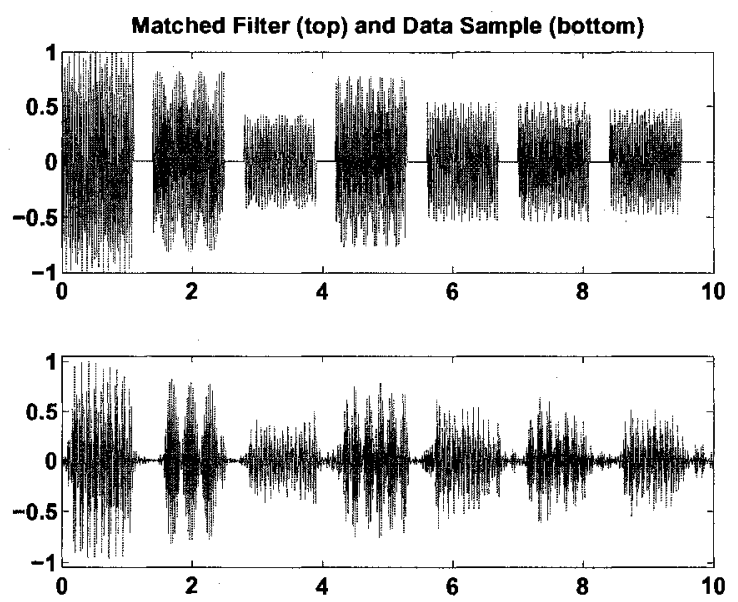

Figure B-3: The resulting matched filter is shown above a recorded pinger transmission for comparison.

Note that the acoustic pulses were recorded at $64 \mathrm{kHz}$ in anticipation of DTAGS having a maximum sample rate of $64 \mathrm{kHz}$ rather than $96 \mathrm{kHz}$. The matched filter was generated at this same sample rate. Sub-pulses having a frequency near the sample rate causes the appearance of amplitude modulation seen in the second sub-pulse in the figure below when 
no explicit amplitude modulation is applied. The matched filter bank was later resampled to $96 \mathrm{kHz}$ to complete processing of the $96 \mathrm{kHz}$ acoustic data collected in this study. 


\section{APPENDIX C}

\section{PINGER CIRCUIT DIAGRAM}

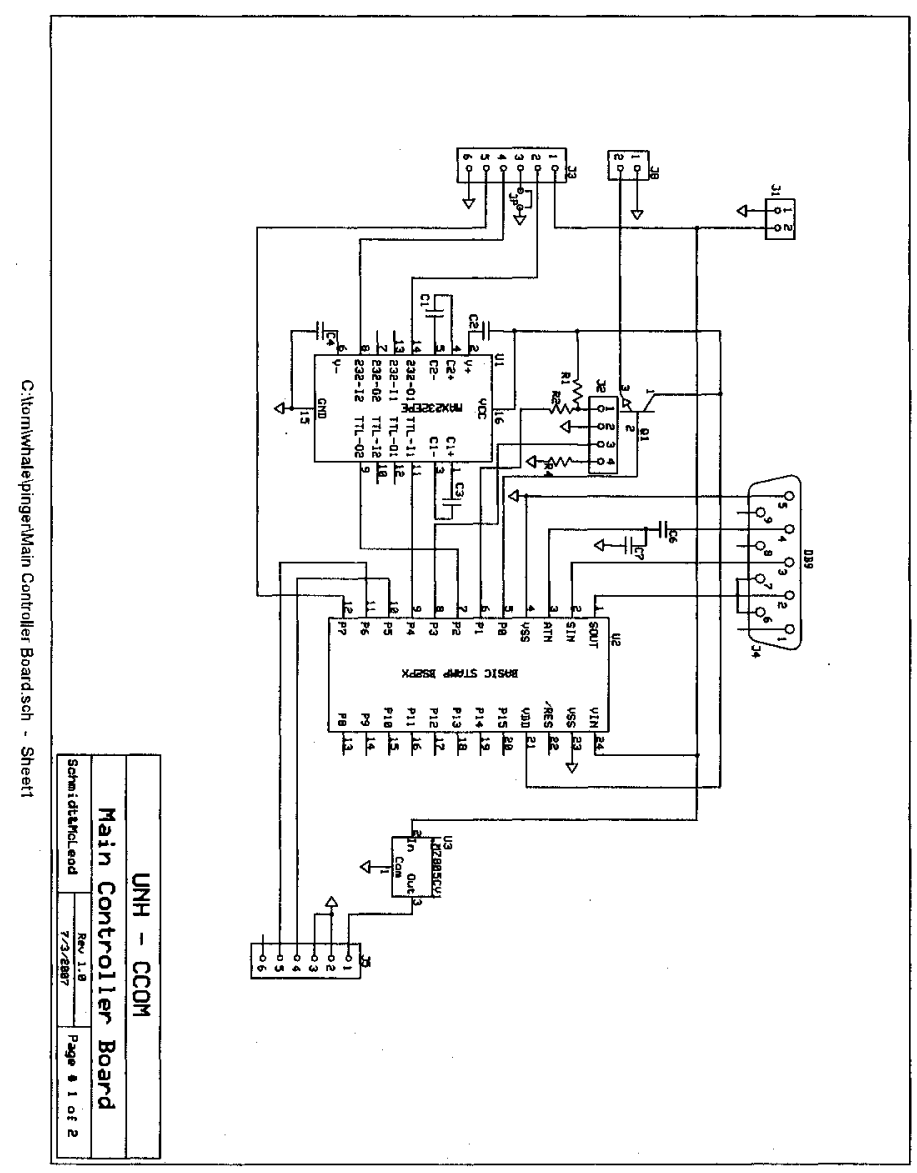

Figure C-1: Pinger circuit diagram. 
APPENDIX D

\section{TIME ENCODING ALGORITHM}

To encode the time into each pinger's acoustic pulse, a single reference permutation of sub-pulses is stored in the pinger's memory. This reference permutation is transmitted for seconds 0-9. For seconds 10-19, an acoustic pulse is generated by skipping adjacent frequency steps in the reference permutation, returning to skipped values when the end of the list is reached. For seconds $20-29$, the acoustic pulse sent is generated by skipping two values in the reference permutation. For seconds 30-39, 40-49 and 50-59, three, four and five steps are skipped respectively. The process is illustrated in Figure D-1 while the actual code can be found in Appendix A. 


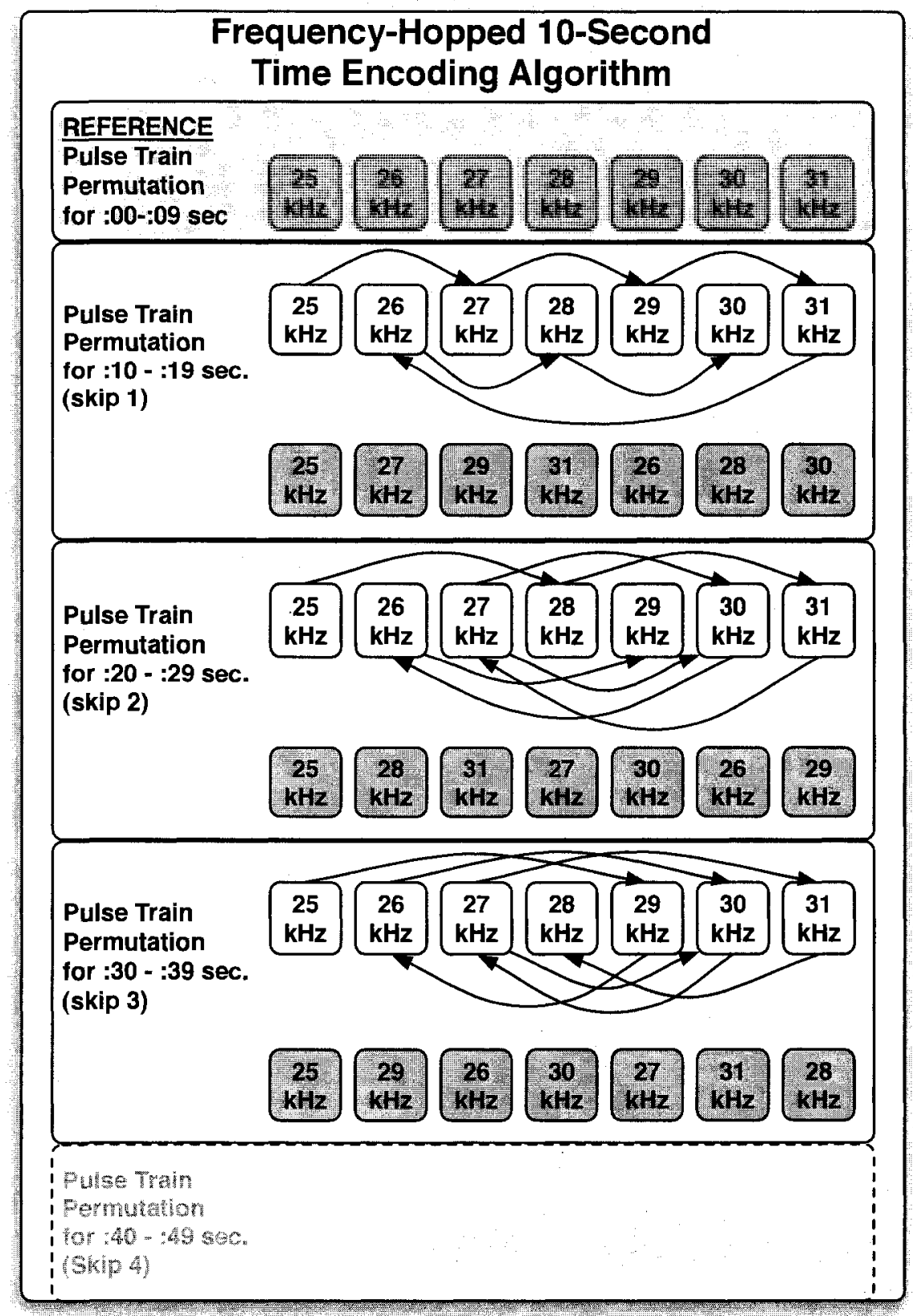

Figure D-1: The method by which time is encoded into each pinger's acoustic signal. 


\section{BIBLIOGRAPHY}

[1] E. L. Hazen, A. Friedlaender, D. Wiley, M. Thompson, C. Ware, and M. T. Weinrich, "Three dimensional prey aggregations and fine scale foraging patterns of humpback whales (Megaptera novaengliae) in Stellwagen Bank Marine Sanctuary," in ICES Annual Meeting, 2007.

[2] - "Oceanographic structuring of fish distribution and fine scale foraging of humpback whales (Megaptera novaeangliae) in Stellwagen Bank Marine Sanctuary," in Society of Marine Mammology, 2007.

[3] A. K. Stimpert, D. Wiley, K. Barton, M. P. Johnson, C. Ware, R. Arsenault, M. Lammers, and W. W. L. Au, "A novel acoustic signal associated with apparent bottom feeding in humpback whales." in Society of Marine Mammals Meeting, San Diego, 2005.

[4] C. Ware, R. Arsenault, M. Plumlee, and D. Wiley, "Visualizing the underwater behavior of humpback whales," IEEE Computer Graphics and Applications, vol. 26, no. 4, pp. $14-18,2006$.

[5] J. H. W. Hain, S. L. Ellis, R. D. Kenney, P. J. Clapham, B. K. Gray, M. T. Weinrich, and I. G. Babb, "Apparent bottom feeding by humpback whales on Stellwagen Bank," Marine Mammal Science, vol. 11, no. 4, pp. 464-479, 1995.

[6] M. P. Johnson and P. L. Tyack, "A digital acoustic recording tag for measuring the response of wild marine mammals to sound," IEEE Journal of Oceanic Engineering, vol. 28 , no. $1,2003$.

[7] W. M. X. Zimmer, M. P. Johnson, A. D'Amico, and P. L. Tyack, "Combining data from a multisensor tag and passive sonar to determine the diving behavior of a sperm 
whale (physeter macrocephalus)," IEEE Journal of Oceanic Engineering, vol. 28, no. 1, pp. $13-28,2003$.

[8] D. H. Cato, R. D. McCauley, and M. Noad, "Potential effects of noise from human activities on marine animals," in Annual Conference of the Australian Acoustical Society, 2004, pp. 369-374.

[9] M. Noad, D. H. Cato, M. D. Stokes, and J. Smith, "Harc - a comprehensive, multiscale colaborative study of migrating humpback whales off the Australian east coast," in Annual Conference of the Australian Marine Sciences Association, 2003, pp. 126127.

[10] M. Noad, R. Dunlop, D. H. Cato, M. D. Stokes, P. Miller, and N. Baiassoni, "Humpback whale social sounds: sources levels and response to playback," in Fourth Joint Meeting of the Acoustical Society of America and Acoustical Society of Japan, vol. 5, Honolulu, HI, 2006, pp. 3012-3012.

[11] S. K. Hooker and R. W. Baird, "Diving and ranging behavior of odontocetes: a methodological review and critique," Mammal Review, vol. 31, no. 1, pp. 81-105, 2001.

[12] G. Kooyman, R. L. Gentry, and D. L. Urquhart, "Northern fur seal diving behavior: A new approach to its study," Science, vol. 193, no. 4251, pp. 411-412, 1976.

[13] S. Fletcher, B. J. Le Boeuf, D. P. Costa, P. L. Tyack, and S. B. Blackwell, "Onboard acoustic recording from diving northern elephant seals," The Journal of the Acoustical Society of America, vol. 100, no. 4, pp. 2531-2539, 1996.

[14] W. C. Burgess, P. L. Tyack, B. J. Le Boeuf, and D. P. Costa, "A programmable acoustic recording tag and first results from free-ranging northern elephant seals," Deep-Sea Reseearch II, vol. 45, pp. 1327-1351, 1998.

[15] J. A. Goldbogen, N. D. Pyenson, and R. E. Shadwick, "Big gulps require high drag for fin whale lunge feeding," Marine Ecology Progress Series, vol. 349, pp. 289-301, 2007. 
[16] J. D. Goodyear, "A sonic/radio tag for monitoring dive depths and underwater movements of whales," The journal of Wildlife Management, vol. 57, no. 3, pp. 503-513, 1993.

[17] P. T. Madsen, P. R., N. U. Kristiansen, M. Wahlberg, I. Kerr, and B. Møhl, "Sperm whale sound production studies with ultrasound time/depth recording tags," The journal of Experimental Biology, vol. 205, pp. 1899-1906, 2002.

[18] A. M. Thode, "Tracking sperm whale (Phyester macrocephalus) dive profiles using a towed passive acoustic array," The Journal of the Acoustical Society of America, vol. 116, no. 1, pp. 245-253, 2004.

[19] C. O. Tiemann, A. M. Thode, J. Straley, V. O'Connell, and K. Folkert, "Threedimensional localization of sperm whales using a single hydrophone," The Journal of the Acoustical Society of America, vol. 120, no. 4, pp. 2355-2365, 2006.

[20] C. O. Tiemann, M. B. Porter, and L. N. Frazer, "Automated model-based localization of marine mammals near hawaii," in OCEANS, 2001, MTS/IEEE Conference and Exhibition, vol. 3, Honolulu, HI, 2001, pp. 1395-1400.

[21] M. Wahlberg, "The acoustic behavior of diving sperm whales observed with a hydrophone array," The Journal of Experimental Marine Biology and Ecology, vol. 281, no. $1-2$, pp. $53-62,2002$.

[22] B. Møhl, M. Wahlberg, and A. Heerfordt, "A large-aperture array of nonlinked receivers for acoustic positioning of biological sound sources," Journal of the Acoustical Society of .America, vol. 109, no. 1, pp. 434-437, 2001.

[23] C. Laplanche, O. Adam, M. Lopatka, and J. Motsch, "Measuring the off-axis angle and the rotational movements of phonating sperm whales using a single hydrophone," Journal of the Acoustical Society of .America, vol. 119, no. 18, pp. 4047-4082, 2006. 
[24] C. O. Tiemann and M. B. Porter, "Localization of marine mammals near hawaii using an acoustic propagation model," The Journal of the Acoustical Society of America, vol. 115, no. 6 , pp. $2834-2843,2004$.

[25] J. H. Miller and D. C. Potter, "Active high frequency phase-array sonar for whale ship-strike avoidance: Target strength measurements", in IEEE/MTS Oceans 2001, Honolulu, HI, 2001, pp. 2104-2107.

[26] I. Lucifredi and P. Stein, "Gray whale target strength measurements and the analysis of backscattered response," Journal of the Acoustical Society of .America, vol. 121, no. 3, pp. 1383-1391, 2007.

[27] J. A. Goldbogen, J. Calambokidis, R. E. Shadwick, E. M. Oleson, M. A. McDonald, and J. A. Hildebrand, "Kinematics of foraging dives and lunge-feeding in fin whales," Journal of Experimental Biology, vol. 209, pp. 1231-1244, 2006.

[28] F. A. Sharpe, "Social foraging of the southeast alaskan humpback whale, Megaptera novaeangliae," Ph.D. dissertation, Simon Fraser University, 2001.

[29] D. A. Helweg, D. S. Houser, and P. W. B. Moore, "An integrated approach to the creation of a humpback whale hearing model," United States Navy, SPAWAR Systems Center, San Diego, CA, Tech. Rep. 1835, 2000.

[30] D. J. Torrieri, Principles of Spread-Spectrum Communications Systems. New York: Springer, 2005.

[31] Citizen, "At-cut crystal unit - cs20 specification sheet," p. 25, 2007. [Online]. Available: http://www.citizen.co.jp/english/crystal/xdcr/CS20.pdf

[32] — "Correlation between crystal cut and freqeuncy vs. temperature characteristics." [Online]. Available: http://www.citizen.co.jp/english/crystal/aspect/index.html

[33] "Garmin gps 16/17 series technical specifications," Garmin International, Inc, Tech. Rep., October, 20052005. 
[34] H. Xue, F. Chai, and N. R. Pettigrew, "A model study of the seasonal circulation in the gulf of maine," Journal of Physical Oceanography, vol. 30, no. 5, pp. 1111-1135, 2000 .

[35] M. Noad and D. H. Cato, "Swimming speeds of singing and non-singing humpback wahles during migration," Marine Mammal Science, vol. 23, no. 2, pp. 481-495, 2007.

[36] J. Brodersen, P. A. Nilsson, J. Ammitzbøll, L.-A. Hansson, C. Skov, and C. Brönmark, "Optimal swimming speed in head currents and effects on distance movement of winter-migrating fish," PLoS ONE, vol. 3, no. 5, p. e2156, May 2008. [Online]. Available: http://dx.doi.org/10.1371\%2Fjournal.pone.0002156 